\section{On the Outage Capacity Distribution of Correlated Keyhole MIMO Channels}

Georgy Levin and Sergey Loyka, Senior Member, IEEE

\begin{abstract}
Keyhole multiple-input-multiple-output (MIMO) channels have recently received significant attention since they can model, to a certain extend, some practically important propagation scenarios and also relay channels in the amplify-and-forward mode. This paper investigates instantaneous signal-to-noise ratio (SNR) and outage capacity distributions of spatially correlated keyhole MIMO channels with perfect channel state information (CSI) at the receive end and with or without CSI at the transmit end. For a small number of antennas, the impact of correlation on the capacity distribution can be characterized by the effective average SNR. This SNR, as well as the outage capacity, decreases with correlation. For a large number of transmit (receive) antennas, the keyhole channel is asymptotically equivalent (in terms of capacity) to the Rayleigh diversity channel with a single transmit (receive) antenna and multiple receive (transmit) antennas. The outage capacity of the keyhole channel is upper-bounded by that of the equivalent Rayleigh diversity channel. When the number of both transmit and receive antennas is large, the outage capacity distribution of the keyhole channel is asymptotically Gaussian. In some cases, the asymptotic Gaussian approximation is accurate already for a reasonably small number of antennas. The perfect transmit CSI is shown to bring a fixed SNR gain. A more general channel model with multiple keyholes is proposed. For a large number of antennas, the capacity of a multikeyhole channel is a normally distributed sum of the capacities of single keyhole channels. The fact that, despite the strong degenerate nature of the keyhole channel, its outage capacity distribution is asymptotically normal indicates that Gaussian distribution has a high degree of universality for the capacity analysis of MIMO channels.
\end{abstract}

Index Terms-Capacity distribution, correlation, keyhole channel, multiple-input-multiple-output (MIMO) system, relay channel, symbol error rate.

\section{INTRODUCTION}

Multiple-input-multiple-output (MIMO) systems have become an attractive solution in wireless communications due to potentially high spectral efficiency. One of the major statistical characteristics of MIMO channels in a multipath environment is their outage capacity, which gives the ultimate upper limit on the error-free information rate with a given probability of outage [1], [35], [2]. The outage capacity distribution of various MIMO channels has been extensively studied, and many analytical and empirical results have been obtained. The uncorrelated and correlated Rayleigh/Rice MIMO channels have been well studied and closed-form expressions for their outage capacity distributions have been found [3]. Many measurement-based works show that a wide range of real channels follows closely those analytical results [4]. Closed-form expressions for the mean capacity and outage capacity distribution of various MIMO channels deploying space-time block coding (STBC) have been also obtained [5], [6].

On the contrary, the outage capacity distribution of keyhole MIMO channels has not been studied in sufficient depth yet. The keyhole channel was analytically predicted in [7] as a channel in multipath

Manuscript received December 6, 2005; revised May 24, 2007. This work was supported in part by the Natural Sciences and Engineering Research Council of Canada. The material in this correspondence was presented in part at the Canadian Workshop on Information Theory, Montreal, QC, Canada, June 2005 and at the IEEE Wireless Communications and Networking Conference, Las Vegas, NV, April 2006.

The authors are with the School of Information Technology and Engineering, University of Ottawa, ON K1N 6N5, Canada (e-mail: glevin@site.uottawa.ca; sergey.loyka@ieee.org).

Communicated by H. Boche, Associate Editor for Communications.

Digital Object Identifier 10.1109/TIT.2008.924721 environment, where certain propagation mechanisms reduce the channel rank. It can be represented as a concatenation of two fading subchannels separated by a keyhole whose dimension is much smaller than the wavelength. The presence of a keyhole degenerates the channel, i.e., its rank is one regardless of the number of transmit (Tx) and receive (Rx) antennas [7]. Thereby, the capacity of such a channel deteriorates significantly compared to the Rayleigh channel with the same number of $\mathrm{Tx}$ and $\mathrm{Rx}$ antennas. There is a significant interest in keyhole channels in recent literature as they can describe some practically important propagation scenarios. Chizhik et al. [8] suggest a keyhole scenario where the only link between Tx and Rx ends is due to the 1-D diffraction. The measurements of the channel capacity along a hallway reported in [9] show a decrease in capacity with distance, which is explained by the keyhole effect in long corridors. The first convincing experimental evidence of a keyhole channel was demonstrated in [10] and [11], where it was shown, in particular, that the keyhole model describes well the wireless channels where the wave propagates via narrow tunnels or waveguides. The importance of a MIMO keyhole channel is also due to its unique position as a channel with only one nonzero eigenmode, which describes the worst case MIMO propagation scenario. It should also be mentioned that relay channels in the amplify-and-forward mode can be represented by the keyhole channel model considered here and, thus, our results apply to such channels as well.

However, the literature dealing with the information theoretic analysis of such channels is rather limited. Closed-form expression for the mean (ergodic) capacity of spatially uncorrelated keyhole channels is presented in [12]. Performance analysis of space-time block codes over uncorrelated keyhole channels is given in [13], where, in particular, the moment generating function of the instantaneous postdetection signal-to-noise ratio (SNR) is derived, and the symbol error rates (SER) for various codes are evaluated. A tight lower bound and an approximation of the mean capacity of spatially correlated keyhole channels are proposed in [14] and [15], respectively.

These papers, however, do not consider the outage capacity, which is a more relevant performance measure in a fading channel from a practical perspective (i.e., for a given quality of service) as compared to the mean capacity. To fill the gap, the present paper derives the closed-form expressions for the instantaneous SNR and the outage capacity distributions of correlated keyhole MIMO channels. We consider a particular but common case where the correlation matrices at the Tx and Rx ends are nonsingular and have distinct eigenvalues. We show that the keyhole channel distribution and so its outage capacity is different from that of traditional diversity channels with single antenna at one end, which also have rank one. However, in terms of capacity and when the number of Tx $(\mathrm{Rx})$ antennas is large, the keyhole channel is asymptotically equivalent to the Rayleigh diversity channel with a single Tx (Rx) antenna. The capacity distribution of the keyhole channel is bounded from below by that of the equivalent Rayleigh diversity channel.

Since the expression for the exact capacity distribution with arbitrary number of Tx and Rx antennas is rather complicated and does not allow for significant insight, we consider two cases, where the number of antennas is either small or asymptotically large. We show that for a small number of antennas, the impact of correlation is characterized by the effective average SNR, i.e., an increase in correlation results in smaller effective SNR and consequently in smaller outage capacity. To derive the outage capacity distribution of keyhole channels with a large number of Tx and Rx antennas, we use the asymptotic analysis, which has been already successfully exploited in [16] and [17] for correlated and uncorrelated Rayleigh-fading MIMO channels, respectively. We show that, under certain mild conditions on correlation and despite the 
degenerate nature of the keyhole channel, its outage capacity distribution is asymptotically Gaussian; while the mean is affected by the average SNR and is independent of correlation, the latter affects the variance. The analogy in asymptotic capacity distribution between the Rayleigh and keyhole MIMO channels indicates that Gaussian approximation has a high degree of universality for MIMO capacity analysis in general. In order to study the impact of the correlation on the outage capacity in explicit form, we propose a scalar measure of MIMO channel correlation and consider as an example the exponential and quadratic exponential correlation matrix models [18], [19], [20] to show analytically that the larger the correlation, the larger the variance of the asymptotic outage capacity. In turn, larger variance results in smaller capacity at low outage probabilities. We demonstrate that in some cases the exact capacity distribution follows closely the asymptotic one already for a reasonably small number of Tx and Rx antennas; the discrepancy is insignificant from the practical point of view. Hence, not only does the simple asymptotic expression offer a significant insight, but can also be applied to realistic problems.

We show that the upper [12] and lower [14] bounds on the mean capacity of keyhole channels can be easily obtained based on the derived instantaneous SNR distribution. Moreover, the upper bound is tight for small SNR and the lower bound is tight for large SNR.

Unlike high-rank MIMO channels with a perfect knowledge of channel state information (CSI) at the Tx end, where finding the capacity is usually associated with significant mathematical complexity, it is shown that in keyhole channels, the perfect Tx CSI brings a fixed SNR gain. Moreover, we find an explicit expression for the optimal input covariance matrix, which shows that the best transmission strategy in this case is the Tx beamforming to the keyhole. Since the fixed SNR gain does not change the channel statistics, we conclude that the results obtained for the keyhole channel with no CSI at the Tx end hold true for the channels with a perfect CSI at both ends as well.

When the channel state information is available at the Rx end only, Alamouti space time code [22] is the optimal transmission technique in keyhole channels with one or two Tx antennas and any number of $\mathrm{Rx}$ antennas, since it achieves the capacity.

As an application of the outage capacity distribution, we demonstrate that a simple yet reasonably accurate estimate of SER in a fading keyhole channel for a variety of modulation formats can be obtained using the outage probability derived from the outage capacity distribution, which becomes especially simple when the number of antennas is large.

Since the ideal keyhole channel in not often encountered in real propagation environment [10], [11], we propose a generalized model of a channel with multiple keyholes, the "multikeyhole channel." We show that asymptotically the capacity of such a channel is a Gaussiandistributed sum of capacities of single keyhole channels. Moreover, while the mean capacity of the multikeyhole channel increases with the number of keyholes, its outage capacity may decrease.

The rest of the paper is organized as follows: In Section II we give basic expressions for the capacity of keyhole channels. The exact distributions of the instantaneous SNR and the outage capacity when CSI is available at the Rx end only are derived in Section III. The asymptotic outage capacity distribution is obtained in Section IV. The mean capacity is discussed in Section V. The capacity of keyhole channels with prefect CSI at the Tx end and capacity achievability are considered in Sections VI and VII, respectively. A SER estimate is proposed in Section VIII. The multikeyhole channel is introduced in Section IX. Section $\mathrm{X}$ concludes the correspondence.

\section{KeYHOLE MIMO CHANNEL CAPACITY}

Consider a spatially correlated keyhole MIMO channel with $n_{t}$ Tx and $n_{r}$ Rx antennas (see Fig. 1). Let element $H_{k m}, k=1 \cdots n_{r} ; m=$ $1 \cdots n_{t}$, of the channel matrix $\mathbf{H}$ be a complex gain from the $m$ th

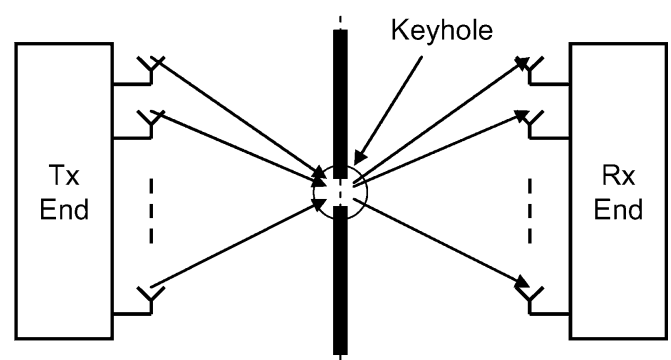

Fig. 1. Keyhole MIMO channel. Each subchannel undergoes Rayleigh fading.

transmit to the $k$ th receive antenna. The keyhole channel matrix is given by [7]

$$
\mathbf{H}=\mathbf{h}_{r} \mathbf{h}_{t}^{H}
$$

where ()$^{H}$ denotes the Hermitian transpose; $\mathbf{h}_{t}\left[n_{t} \times 1\right]$ and $\mathbf{h}_{r}\left[n_{r} \times 1\right]$ are the vectors representing the complex gains from the transmit antennas to the keyhole and from the keyhole to the receive antennas, respectively. Assume that $\mathbf{h}_{t}$ and $\mathbf{h}_{r}$ are mutually independent complex circular symmetric correlated Gaussian random vectors with zero means and correlation matrices $\mathbf{R}_{t}=E\left\{\mathbf{h}_{t} \mathbf{h}_{t}^{H}\right\}$ and $\mathbf{R}_{r}=E\left\{\mathbf{h}_{r} \mathbf{h}_{r}^{H}\right\}$, respectively, where $E\{\}$ denotes the expectation. Without loss of generality, $\mathbf{H}$ is normalized so that $E\left\{\|\mathbf{H}\|^{2}\right\}=n_{t} n_{r}$, where $\|\mathbf{H}\|=\sqrt{\operatorname{tr}\left\{\mathbf{H H}^{H}\right\}}$ is the $L_{2}$ norm, and $n_{t}^{-1} E\left\{\left\|\mathbf{h}_{t}\right\|^{2}\right\}=n_{r}^{-1} E\left\{\left\|\mathbf{h}_{r}\right\|^{2}\right\}=1$, which also implies $n_{t}^{-1} \operatorname{tr}\left\{\mathbf{R}_{t}\right\}=n_{r}^{-1} \operatorname{tr}\left\{\mathbf{R}_{r}\right\}=1$.

When the CSI is available at the Rx end but not the Tx end, the instantaneous capacity (i.e., the capacity of a given channel realization) of a frequency-flat quasi-static MIMO channel in natural units [nat] is given by [2]

$$
C=\ln \operatorname{det}\left(\mathbf{I}+\frac{\gamma_{0}}{n_{t} n_{r}} \mathbf{H} \mathbf{H}^{H}\right)
$$

where det is the determinant, $\mathbf{I}$ is $n_{r} \times n_{r}$ identity matrix, and $\gamma_{0}$ is the total average SNR at the Rx end. Substituting (1) in (2) and using the fact that for any matrices $\mathbf{A}$ and $\mathbf{B}$ with suitable dimensionality $\operatorname{det}[\mathbf{I}+\mathbf{A B}]=\operatorname{det}[\mathbf{I}+\mathbf{B A}]$ [1], [35], it is straightforward to show that the instantaneous capacity of the keyhole channel is [21]

$$
C=\ln \left(1+\frac{\gamma_{0}}{n_{t} n_{r}} \alpha\right)
$$

where $\alpha=\left\|\mathbf{h}_{t}\right\|^{2}\left\|\mathbf{h}_{r}\right\|^{2}$ is the power gain of the equivalent scalar channel. Up to a constant factor, $\alpha$ determines the instantaneous $\operatorname{SNR}\left(=\alpha \cdot \gamma_{0} /\left(n_{t} n_{r}\right)\right)$ in the equivalent scalar channel. Since the instantaneous capacity is a continuous, monotonically increasing function of $\alpha$, the cumulative distribution function (cdf) of $C$, which is also the outage capacity distribution $F_{C}(x)$, is given by

$$
F_{C}(x)=F_{\alpha}\left(\frac{n_{t} n_{r}}{\gamma_{0}}\left(e^{x}-1\right)\right)
$$

where $F_{\alpha}(x)$ is the cdf of $\alpha$. The exact expression for $F_{\alpha}(x)$ is given and analyzed in the next section.

\section{SNR AND OUTAGE CAPACITY DISTRIBUTION}

Theorem 1: Let $\alpha=\beta_{t} \cdot \beta_{r}$, where $\beta_{t}=\left\|\mathbf{h}_{t}\right\|^{2}, \beta_{r}=\left\|\mathbf{h}_{r}\right\|^{2}$ and $\mathbf{h}_{t}, \mathbf{h}_{r}$ are mutually independent complex circular symmetric correlated Gaussian random vectors. When both $\mathbf{R}_{t}$ and $\mathbf{R}_{r}$ are nonsingular and have distinct eigenvalues $\lambda_{k}^{t}, k=1 \cdots n_{t}$, and $\lambda_{m}^{r}, m=1 \cdots n_{r}$, 
respectively, the probability density function (pdf) $f_{\alpha}(z)$ and the cdf $F_{\alpha}(z)$ of $\alpha$ are as follows:

$$
\begin{aligned}
f_{\alpha}(x)= & 2 \sum_{k=1}^{n_{t}} \sum_{m=1}^{n_{r}} \frac{A_{k}^{t} A_{m}^{r}}{\lambda_{k}^{t} \lambda_{m}^{r}} K_{0}\left(\sqrt{\frac{4 x}{\lambda_{k}^{t} \lambda_{m}^{r}}}\right), x \geq 0 \\
F_{\alpha}(x)= & 1-\sum_{k=1}^{n_{t}} \sum_{m=1}^{n_{r}} A_{k}^{t} \\
& \times A_{m}^{r} \sqrt{\frac{4 x}{\lambda_{k}^{t} \lambda_{m}^{r}}} K_{1}\left(\sqrt{\frac{4 x}{\lambda_{k}^{t} \lambda_{m}^{r}}}\right), x \geq 0
\end{aligned}
$$

where $K_{n}(x)$ is the $n$-order modified Bessel function of the second kind, $A_{k}^{t}$ and $A_{m}^{r}$ are the coefficients of a partial fraction decomposition given by

$$
A_{k}=\prod_{\substack{m=1 \\ m \neq k}}^{n}\left(1-\frac{\lambda_{m}}{\lambda_{k}}\right)^{-1}
$$

and $A_{k}$ is either $A_{k}^{t}$ or $A_{k}^{r}, \lambda_{k}$ is either $\lambda_{k}^{t}$ or $\lambda_{k}^{r}$, and $n$ is either $n_{t}$ or $n_{r}$.

Proof: See Appendix A.

Consider a number of cases where Theorem 1 does not apply or applies with some modifications.

i) The channel at the $\mathrm{Tx}, \mathrm{Rx}$, or both ends is uncorrelated, i.e., $\lambda_{k}=1$ for $k=1 \cdots n$ (the eigenvalues are not distinct). While Theorem 1 does not apply in this case, $f_{\alpha}(x)$ and $F_{\alpha}(x)$ can be evaluated based on the characteristic function $(\mathrm{CF}) \Phi_{\alpha}(\omega)$ given in [12] for uncorrelated keyhole channels.

ii) There is a number of fully correlated antennas, i.e., $\mathbf{R}_{t}, \mathbf{R}_{r}$ or both have some zero eigenvalues. In this case the pdf and so cdf of $\alpha$ is also calculated by (5) and (6), respectively, but the summation is taken over nonzero eigenvalues only, i.e., zero eigenvalues and corresponding eigenvectors are excluded as they do not contribute to the SNR. This follows directly from the proof of Theorem 1. In particular, when the channel is fully correlated at both the Tx and Rx ends, it is equivalent to a keyhole channel with a single antenna at each end and the gains equal to $n_{t}$ and $n_{r}$, respectively. In this case, $A_{1}^{t}=A_{1}^{r}=1, \lambda_{1}^{t}=n_{t}$ and $\lambda_{1}^{r}=n_{r}$, so that (5) and (6) reduce to

$$
\begin{aligned}
& f_{\alpha}(x)=\frac{2}{n_{t} n_{r}} K_{0}\left(\sqrt{\frac{4 x}{n_{t} n_{r}}}\right), x \geq 0 \\
& F_{\alpha}(x)=1-\sqrt{\frac{4 x}{n_{t} n_{r}}} K_{1}\left(\sqrt{\frac{4 x}{n_{t} n_{r}}}\right) ; x \geq 0 .
\end{aligned}
$$

In all the cases, the exact expression for the outage capacity distribution is obtained from (4) using the instantaneous SNR cdf.

The analytical expressions above have been validated by MonteCarlo simulations for various $n_{t}, n_{r}, \gamma_{0}, \mathbf{R}_{t}$ and $\mathbf{R}_{r}$. In all considered cases, no difference has been found between analytical and numerical results.

To get some insight into (4), let us consider a $2 \times 2$ keyhole MIMO channel with equal correlation matrices

$$
\mathbf{R}_{t}=\mathbf{R}_{r}=\left[\begin{array}{cc}
1 & r \\
r^{*} & 1
\end{array}\right], \quad|r|<1
$$

where $r^{*}$ is a complex conjugate of $r$. Using the Maclaurin series of $x \cdot K_{1}(x)$ and $\exp (x)$, it is straightforward to show that for small $x$

$$
F_{C}(x) \geq c \cdot\left[\frac{x}{\gamma_{0}\left(1-|r|^{2}\right)}\right]^{2}
$$

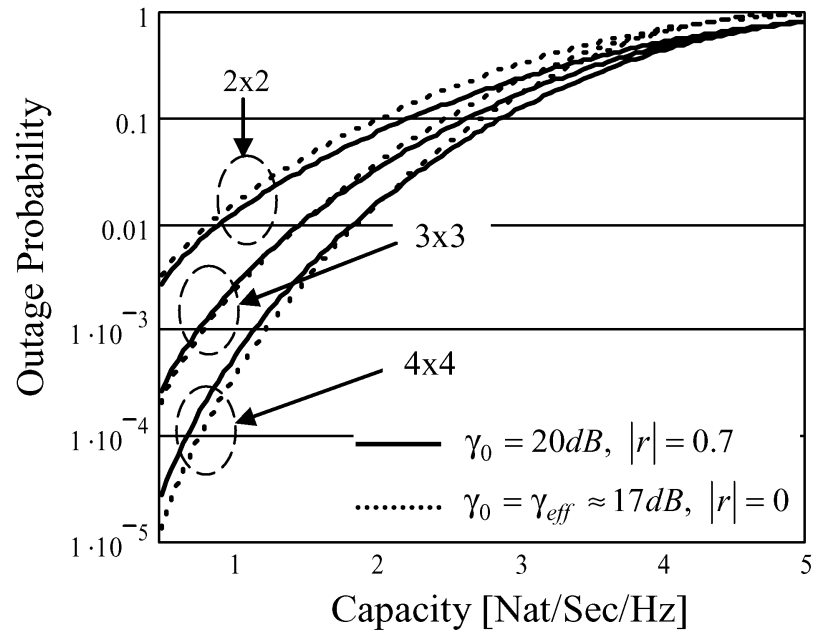

Fig. 2. Outage capacity distribution: correlated keyhole channel (bold line), uncorrelated one with effective average SNR (dotted line).

where $c=5-4\left(\gamma_{e}-\ln (2)\right)$ and $\gamma_{e} \approx 0.577$ is the Euler constant [24]. Using numerical simulations, we found that the lower bound in (11) gives a good approximation for the capacity distribution at outage probabilities $F_{C}(x)>10^{-7}$. Moreover, from (11), an effective average SNR, i.e., the SNR of an uncorrelated keyhole channel with the same probability of outage, can be defined as

$$
\gamma_{\text {eff }}=\gamma_{0}\left(1-|r|^{2}\right)
$$

Apparently, an increase in correlation decreases the effective average SNR and results in lower capacity. For example, $|r|=0.7$ corresponds to $\mathrm{a} \approx 3 \mathrm{~dB}$ decrease in $\gamma_{\mathrm{eff}}$. Interestingly, (12) is identical to the effective SNR in the correlated Rayleigh channels with maximum ratio combining (MRC) [23] and in full-rank MIMO channels [18]. From numerical simulations, the impact of correlation on the outage capacity is accurately characterized by the effective average SNR not only for $2 \times 2$ keyhole channels, but also for channels with up to four antennas at each end. To demonstrate this, Fig. 2 compares the outage capacity distribution of $2 \times 2,3 \times 3$, and $4 \times 4$ correlated keyhole channels to that of the equivalent (with respect to effective average SNR) uncorrelated keyhole channels. For the correlated channels, $\mathbf{R}_{t}$ and $\mathbf{R}_{r}$ are simulated using exponential correlation model [18] with the same correlation parameter $|r|=0.7$ at both Tx and Rx ends. The average SNR at the uncorrelated channels is set to $\gamma_{\text {eff }}$ given by (12). The difference between the capacity distributions of correlated and corresponding uncorrelated channels increases with the number of antennas. Similar observations can also be made for the channels where $\mathbf{R}_{t}$ and $\mathbf{R}_{r}$ are modeled by quadratic exponential correlation matrices ${ }^{1}$ [19], [20].

For more than $4 \times 4$ systems, the effective SNR alone is not enough to represent the effect of correlation. In this case (4) has a complicated form, which makes it difficult to obtain insight and to evaluate the effect of various parameters on the capacity. In particular, the effect of correlation is difficult to see. Moreover, when $n_{t}$ or $n_{r}$ are large and the correlation at the Tx or Rx ends is low, the partial fraction decomposition coefficients in (7) become too large, so that numerical evaluation of (4) suffers from the loss of precision. To overcome these problems, we derive below the asymptotic outage capacity distributions when $n_{t}, n_{r}$ or both are asymptotically large.

${ }^{1}$ The exponential and quadratic exponential correlation models are considered in detail in Section IV. The corresponding correlation matrices are given by $(21)$ and $(25)$, respectively. 


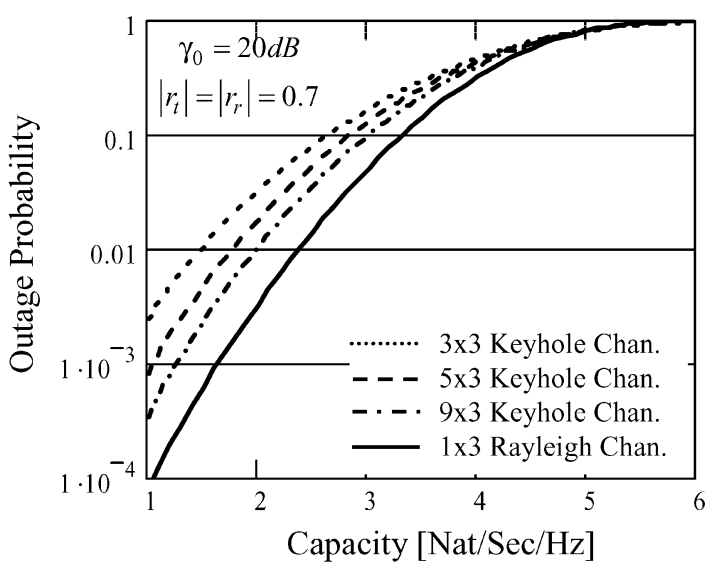

Fig. 3. Outage capacity distributions: $n_{t} \times 3$ keyhole and $1 \times 3$ Rayleigh correlated channels.

\section{ASYMPTOTIC OUTAGE CAPACITY DISTRIBUTIONS}

Theorem 2: Consider an $n_{t} \times n_{r}$ keyhole channel, where $\mathbf{h}_{t}$ and $\mathbf{h}_{r}$ are mutually independent complex circular symmetric correlated Gaussian random vectors with zero mean and correlation matrices $\mathbf{R}_{t}$ and $\mathbf{R}_{r}$.

i) If $\lim _{n_{t} \rightarrow \infty} n_{t}^{-1} \operatorname{tr}\left\{\mathbf{R}_{t}\right\}<\infty$ and $\lim _{n_{t} \rightarrow \infty} n_{t}^{-1}\left\|\mathbf{R}_{t}\right\|=0$, then there is an asymptotically equivalent $1 \times n_{r}$ Rayleigh fading channel, so that the instantaneous capacities of both the keyhole and Rayleigh channels are identical in distribution as $n_{t} \rightarrow \infty$, i.e.,

$$
C \stackrel{p}{\rightarrow} \ln \left(1+\frac{\gamma_{0}}{n_{r}} \beta_{r}\right) \text { as } n_{t} \rightarrow \infty
$$

where $\stackrel{p}{\rightarrow}$ denotes convergence in probability.

ii) Due to the symmetry in (3) and under the same conditions, (13) holds true as $n_{r} \rightarrow \infty$, if Tx and Rx ends are exchanged.

Proof: See Appendix B.

The asymptotic behavior of the keyhole channel indicated by Theorem 2 is well explained by the fact that when $n_{t}\left(n_{r}\right) \rightarrow \infty$, the Rayleigh subchannel (see Fig. 1) at the Tx (Rx) end is asymptotically a nonfading AWGN one with a single equivalent $\mathrm{Tx}(\mathrm{Rx})$ antenna, and the whole keyhole channel is equivalent to the $\mathrm{Rx}(\mathrm{Tx})$ diversity Rayleigh channel. This observation has already been made in [13] for uncorrelated keyhole channels. Theorem 2 shows that the same is true for the correlated keyhole channels under the aforementioned conditions. $^{2}$

Corollary 2.1: If $\mathbf{R}_{r}$ is nonsingular and has distinct eigenvalues, the asymptotic outage capacity distribution of the keyhole channel is given by

$$
F_{C}(x) \rightarrow F_{\beta_{r}}\left(\frac{n_{r}}{\gamma_{0}}\left(e^{x}-1\right)\right) \text { as } n_{t} \rightarrow \infty
$$

where

$$
F_{\beta_{r}}(x)=1-\sum_{k=1}^{n_{r}} A_{k}^{r} \exp \left\{-x / \lambda_{k}\right\}, \quad x \geq 0
$$

Due to the symmetry in (3) and under the same conditions, (14) holds true as $n_{r} \rightarrow \infty$, if Tx and Rx ends are exchanged.

Proof: See Appendix B. [27].

${ }^{2}$ A physical interpretation to the conditions of Theorem 2 can be found in
Consider the outage capacity distribution when the number of antennas increases. Assume that new antennas do not change the electromagnetic environment and thereby the channel for already existing ones. In such a case, an increase in the number of Tx antennas has two opposite effects: 1) under a total Tx power constraint, it decreases the average transmitted power per Tx antenna, and 2) increases the diversity order of the channel. At low outage probabilities the effect of the increasing diversity order is dominant, which causes $F_{C}(x)$ to decrease. At high outage probabilities, the effect of decreasing Tx power prevails over the increasing diversity order so that $F_{C}(x)$ increases. Thus, the cdf curves of two keyhole channels with $n$ and $m(m>n)$ Tx antennas and a fixed (same) number of Rx antennas should cross each other, so that at low outage probabilities the channel with $m$ antennas has higher outage capacity and, correspondently, the channel with $n$ antennas has higher outage capacity at high outage probabilities. Since the equivalent Rayleigh diversity channel is an asymptotic case of the keyhole channel as $n_{t} \rightarrow \infty$ the following inequality holds at low outage probabilities

$$
F_{C}^{R}(x) \leq F_{C}^{K}(x)
$$

where $F_{C}^{K}(x)$ and $F_{C}^{R}(x)$ are the capacity distributions of the keyhole and equivalent Rayleigh diversity channels, respectively, i.e., at low outage probabilities, the outage capacity of the equivalent Rayleigh channel upper-bounds that of the keyhole one. To validate (16), the capacity distributions of correlated $n_{t} \times 3$ keyhole and equivalent $1 \times 3$ Rayleigh diversity channels are plotted in Fig. 3. The exponential correlation model [18] with the correlation parameter $|r|=0.7$ was used to simulate both $\mathbf{R}_{t}$ and $\mathbf{R}_{r}$. Clearly, the outage capacity of the Rayleigh channel is higher than that of the keyhole one, the latter approached the former as $n_{t}$ increases.

Even though Theorem 2 shows the relationship between the keyhole channel and the equivalent Rayleigh diversity channels, the asymptotic distribution in (14) is still complicated and does not contribute much to the understanding of the impact of various parameters in general and correlation in particular on the outage capacity. To gain such understanding, we proceed with the following theorem.

Theorem 3: Let $C$ be the instantaneous capacity of the correlated keyhole channel defined in (2). When both $n_{t}$ and $n_{r}$ tend to infinity, the distribution of $C$ is asymptotically Gaussian if $\lim _{n_{t} \rightarrow \infty} n_{t}^{-1} \operatorname{tr}\left\{\mathbf{R}_{t}\right\}<\infty, \lim _{n_{r} \rightarrow \infty} n_{r}^{-1} \operatorname{tr}\left\{\mathbf{R}_{r}\right\}<\infty$ and $\lim _{n_{t} \rightarrow \infty} n_{t}^{-2}\left\|\mathbf{R}_{t}\right\|^{2}=\lim _{n_{r} \rightarrow \infty} n_{r}^{-2}\left\|\mathbf{R}_{r}\right\|^{2}=0$. Moreover, if $\mathbf{h}_{t}$ and $\mathbf{h}_{r}$ are normalized so that $\lim _{n_{t} \rightarrow \infty} n_{t}^{-1} \operatorname{tr}\left\{\mathbf{R}_{t}\right\}=$ $1, \lim _{n_{r} \rightarrow \infty} n_{r}^{-1} \operatorname{tr}\left\{\mathbf{R}_{r}\right\}=1$, the mean $\mu$ and the variance $\sigma^{2}$ of $C$ are as follows:

$$
\begin{aligned}
\mu & =\ln \left(1+\gamma_{0}\right) \\
\sigma^{2} & =\left(\frac{\gamma_{0}}{1+\gamma_{0}}\right)^{2}\left(\frac{1}{n_{t}^{2}}\left\|\mathbf{R}_{t}\right\|^{2}+\frac{1}{n_{r}^{2}}\left\|\mathbf{R}_{r}\right\|^{2}\right) .
\end{aligned}
$$

\section{Proof: See Appendix C.}

Note that the conditions of Theorem 3 do not require distinct eigenvalues of the correlation matrices $\mathbf{R}_{t}$ and $\mathbf{R}_{r}$. Hence, the outage capacity distribution of the uncorrelated keyhole MIMO channel with $\mathbf{R}_{t}=\mathbf{R}_{r}=\mathbf{I}$ is asymptotically normal, with $\mu=\ln \left(1+\gamma_{0}\right)$ and $\sigma^{2}=\left(\gamma_{0} /\left(1+\gamma_{0}\right)\right)^{-2}\left(n_{t}^{-1}+n_{r}^{-1}\right)$. From (17), it is not always true that an increase in the number of antennas decreases the variance and thereby the outage probability (as one would intuitively expect based on the increasing diversity argument), but only if $\sigma^{2}$ is monotonically decreasing with $n_{t}$ and $n_{r}$, i.e., if $\left\|\mathbf{R}_{t}\right\|$ and $\left\|\mathbf{R}_{r}\right\|$ increase not faster than $n_{t}^{1-\varepsilon_{1}}$ and $n_{r}^{1-\varepsilon_{2}}$, respectively, for some $\varepsilon_{1}, \varepsilon_{2}>0$. Even though the conditions of Theorem 3 do not require such monotonicity, we show 
below that the exponential [18] and quadratic exponential [19], [20] correlation models possess this monotonicity property.

Since $\mu$ is a function of $\gamma_{0}$ only, the effect of correlation on the mean capacity vanishes asymptotically. In contrast, the variance $\sigma^{2}$ is affected by the correlation, but does not depend on the average SNR from moderate to high $\gamma_{0}$, since $\gamma_{0} /\left(1+\gamma_{0}\right) \approx 1$ in (17). This explains why for large $n_{t}$ and $n_{r}$ the effect of correlation is not adequately represented by the effective average SNR in (12). Note also that the asymptotic mean capacity in (17) is identical to the upper bound on the mean capacity of the finite order uncorrelated keyhole channel given in [12], i.e., Theorem 3 shows that the bound also holds for the correlated channels and it is asymptotically tight.

To analyze $\sigma^{2}$ in (17), let $\mathbf{R} \in \Re$, where $\Re$ is a set of all $n \times n$ correlation matrices such that $\operatorname{tr}(\mathbf{R})=n$. Using Cauchy-Schwarz's inequality

$$
\frac{1}{n^{2}}\|\mathbf{R}\|^{2}=\frac{1}{n^{2}} \sum_{k=1}^{n} \lambda_{k}^{2} \geq \frac{1}{n^{2}}\left(\frac{1}{\sqrt{n}} \sum_{k=1}^{n} \lambda_{k}\right)^{2}=\frac{1}{n}
$$

with the equality if $\lambda_{k}=\lambda_{m}$ for all $k, m=1 \ldots n$, where $\lambda_{k}$ are eigenvalues of $\mathbf{R}$, i.e., if $\mathbf{R}=\mathbf{I}$. Thus, $n^{-2}\|\mathbf{R}\|^{2}$ achieves its minimum when the channel at the $\mathrm{Tx}(\mathrm{Rx})$ end is uncorrelated with the same power at each $\operatorname{Tx}(\mathrm{Rx})$ antenna. Furthermore, since every $\mathbf{R} \in \Re$ is positive semi-definite $\left(\lambda_{k} \geq 0, k=1 \ldots n\right)$

$$
\frac{1}{n^{2}}\|\mathbf{R}\|^{2}=\frac{1}{n^{2}} \sum_{k=1}^{n} \lambda_{k}^{2} \leq \frac{1}{n^{2}}\left(\sum_{k=1}^{n} \lambda_{k}\right)^{2}=1
$$

with the equality if $\lambda_{k}=n$ for some $k$, and $\lambda_{m}=0 \forall m \neq k$. Thus, $n^{-2}\|\mathbf{R}\|^{2}$ achieves its maximum when the channel at the Tx $(\mathrm{Rx})$ end is fully correlated. From (18), (19) and following the properties of the $L_{2}$ norm [24], $n^{-2}\|\mathbf{R}\|^{2}$ is a mapping of $\Re$ onto a closed interval of real numbers $[1 / n ; 1]$ (note that $[1 / n ; 1]$ converges to $(0 ; 1]$ as $n \rightarrow$ $\infty$, which has a certain degree of similarity with the scalar correlation coefficient). This leads to the following definition.

Definition 1: A channel with correlation matrix $\mathbf{R}_{1} \in \Re$ is said to be equally or more correlated than one with $\mathbf{R}_{2} \in \Re$ if

$$
n^{-1}\left\|\mathbf{R}_{1}\right\| \geq n^{-1}\left\|\mathbf{R}_{2}\right\|
$$

Definition 1 introduces $n^{-2}\|\mathbf{R}\|^{2}$ as a measure of correlation for channels with large $n$, alternative to that in [25]. Unlike [25], Definition 1 is not based on the majorization theory [26] and allows comparing correlations between any pair $\mathbf{R}_{1}, \mathbf{R}_{2} \in \Re$, with no exception. ${ }^{3}$ Equation (17) shows that the variance of outage capacity is directly proportional to the total measure of correlations $\left(n_{t}^{-2}\left\|\mathbf{R}_{t}\right\|^{2}+n_{r}^{-2}\left\|\mathbf{R}_{r}\right\|^{2}\right)$. As a simple application of this definition and Theorem 3 , we have the following result.

Corollary 3.1: Consider two keyhole channels with the same $n_{t}, n_{r}, \gamma_{0}$ and with different variances $\sigma_{1}^{2}>\sigma_{2}^{2}$ of instantaneous capacity (see (17)),.i.e., according to Definition 1 , the channel with $\sigma_{1}^{2}$ has higher correlation. Thus, $F_{C}^{1}(x)>F_{C}^{2}(x)$ for $x>\mu$, and $F_{C}^{1}(x)<F_{C}^{2}(x)$ for $x>\mu$, i.e., the channel with higher correlation has smaller outage capacity (higher outage probability) at outage region $F_{C}(x)<1 / 2$, and larger outage capacity at outage region $F_{C}(x)>1 / 2$; the latter region, however, has limited importance from practical perspective).

Proof: Under the conditions of Theorem 3, the outage capacity of both channels is asymptotically Gaussian with equal means $\mu_{1}=\mu_{2}=$ $\mu$. Compare two equal-mean Gaussian cdfs $F_{C}^{1}(x)$ (with variance $\sigma_{1}^{2}$ )

\footnotetext{
${ }^{3}$ The majorization-theory-based definition does not allow full ordering of correlation matrices with more than two nonzero eigenvalues [25], i.e., some correlation matrices cannot be compared.
}

and $F_{C}^{2}(x)$ (with variance $\sigma_{2}^{2}$ ). It follows that they cross each other at the single point $x=\mu$ such that $F_{C}^{1}(\mu)=F_{C}^{2}(\mu)=1 / 2$, and that $F_{C}^{1}(x)>F_{C}^{2}(x)$ for $x<\mu$, and $F_{C}^{1}(x)<F_{C}^{2}(x)$ for $x>\mu$.Q.E.D.

To validate the general discussion above and to show explicitly the impact of correlation on asymptotic outage capacity distribution, consider two single-parameter correlation matrix models for $\mathbf{R}_{t}$ and $\mathbf{R}_{r}$.

\section{A. Exponential Correlation Model}

In this model the elements of correlation matrix $\mathbf{R}$, either $\mathbf{R}_{t}$ or $\mathbf{R}_{r}$, are represented through a single complex correlation parameter $r$, which is the correlation between adjacent antennas [18]

$$
\mathbf{R}_{k m}=\left\{\begin{array}{l}
r^{m-k} ; m \geq k \\
\left(r^{*}\right)^{k-m} ; m<k
\end{array},|r|<1\right.
$$

This model allows for significant insight and has been successfully used for many communications problems. Despite its simplicity, it is a physically reasonable model in the sense that the correlation decreases as the distance between antennas increases. It can be shown that $\mathbf{R}$ in (21) satisfies the conditions of Theorem 3, i.e., $n^{-1} \operatorname{tr}\{\mathbf{R}\}=1$ and as $n \rightarrow \infty$

$$
n^{-2}\|\mathbf{R}\|^{2} \rightarrow \frac{1}{n} \cdot \frac{1+|r|^{2}}{1-|r|^{2}} \rightarrow 0,|r|<1
$$

(see Appendix D for a proof). Thus, when both $\mathbf{R}_{t}$ and $\mathbf{R}_{r}$ are given by the exponential model, the outage capacity distribution of such a keyhole channel is asymptotically Gaussian with the mean given in (17) and the variance

$$
\sigma^{2}=\left(\frac{\gamma_{0}}{1+\gamma_{0}}\right)^{2}\left(\frac{1}{n_{t}} \cdot \frac{1+\left|r_{t}\right|^{2}}{1-\left|r_{t}\right|^{2}}+\frac{1}{n_{r}} \cdot \frac{1+\left|r_{r}\right|^{2}}{1-\left|r_{r}\right|^{2}}\right)
$$

where $r_{t}$ and $r_{r}$ are the correlation parameters in $\mathbf{R}_{t}$ and $\mathbf{R}_{r}$, respectively. Note that $\sigma^{2}$ is monotonically decreasing with $n_{t}$ and $n_{r}$ (monotonicity property). To get some insight, assume that $n=n_{t}=n_{r}$ and $r=r_{t}=r_{r}$; then, for the same $\gamma_{0}$ in both channels, the capacity distributions of uncorrelated $r=0$ and correlated $r \neq 0$ channels have the same mean $\mu=\ln \left(1+\gamma_{0}\right)$, but different variances $\sigma_{u}^{2}$ and $\sigma_{c}^{2}$, so that

$$
\eta=\frac{\sigma_{c}^{2}}{\sigma_{u}^{2}}=\frac{1+|r|^{2}}{1-|r|^{2}} \geq 1,|r|<1 .
$$

From (24), $\sigma_{c}^{2} \geq \sigma_{u}^{2}$, and $\eta$ is a monotonically increasing function of $|r|$, i.e., the larger $|r|$ results in larger $\sigma_{c}^{2}$. This supports Definition 1 . Thus, following the general discussion above, the outage capacity of the uncorrelated asymptotic channel is larger than that of the correlated one at outage probabilities less than 0.5 . Note that even for small $|r|$, the capacity gap between correlated and uncorrelated channels can still be significant at low outage probabilities. As an example, Fig. 4 shows the asymptotic outage capacity distributions of the $3 \times 3$ keyhole channels with exponential correlation at both ends for $|r|=\left|r_{t}\right|=\left|r_{r}\right|$. Clearly, the outage capacity decreases at outage probabilities less than 0.5 as $|r|$ increases. For $|r| \leq 0.2$, correlation has no significant impact on the asymptotic capacity except at extremely low outage probabilities.

\section{B. Quadratic Exponential ( $Q E)$ Model}

This is a physically based single-parameter correlation matrix model where the elements of the correlation matrix $\mathbf{R}$ are given by [19], [20]

$$
\mathbf{R}_{k m}=\left\{\begin{array}{l}
r^{(m-k)^{2}} ; m \geq k \\
\left(r^{*}\right)^{(k-m)^{2}} ; m<k
\end{array},|r|<1 .\right.
$$

Here the correlation between different antennas decays much faster with distance $|m-k|$ than in the previous example. This is a physical 


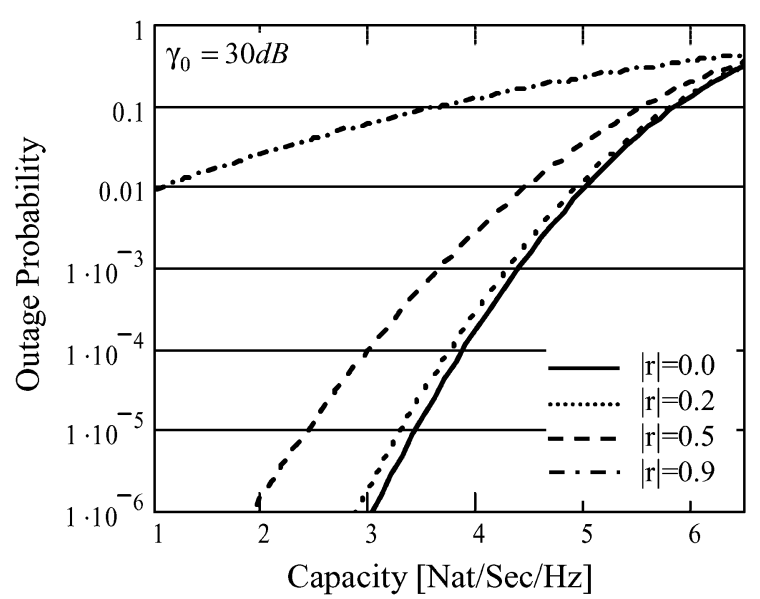

Fig. 4. Asymptotic outage capacity distributions of $3 \times 3$ keyhole channels with exponential correlation.

model as it represents the scenario with a Gaussian profile of multipath angle-of-arrival [19], [20]. Similarly to the exponential model, the QE one also satisfies the conditions of Theorem 3, i.e., $n^{-1} \operatorname{tr}\{\mathbf{R}\}=1$ and for a sufficiently large $n$ and $|r|<1$

$$
\frac{1}{n} \cdot\left[1+\frac{\sqrt{\pi}}{\nu(r)} \mathcal{E}[\nu(r)]\right] \leq n^{-2}\|\mathbf{R}\|^{2} \leq \frac{1}{n} \cdot\left[1+\frac{\sqrt{\pi}}{\nu(r)}\right]
$$

where $\nu(r)=\sqrt{-2 \ln |r|}, \mathcal{E}(x)=\operatorname{erfc}(x)$ is the complementary error function [24] (see Appendix D for a proof). Since both the upper and lower bounds converge asymptotically to zero, then $\lim _{n \rightarrow \infty} n^{-2}\|\mathbf{R}\|^{2}=0$. Therefore, from Theorem 3, the outage capacity distribution of the keyhole channel with QE correlation at both the Tx and Rx ends is asymptotically Gaussian with the mean $\mu=\ln \left(1+\gamma_{0}\right)$ and the variance bounded for $\left|r_{t}\right|,\left|r_{r}\right|<1$ by

$$
\begin{aligned}
\sigma^{2} \leq & \left(\frac{\gamma_{0}}{1+\gamma_{0}}\right)^{2}\left(\frac{1}{n_{t}}\left[1+\frac{\sqrt{\pi}}{\nu\left(r_{t}\right)}\right]\right. \\
& \left.+\frac{1}{n_{r}}\left[1+\frac{\sqrt{\pi}}{\nu\left(r_{r}\right)}\right]\right) \\
\sigma^{2} \geq & \left(\frac{\gamma_{0}}{1+\gamma_{0}}\right)^{2}\left(\frac{1}{n_{t}}\left[1+\frac{\sqrt{\pi}}{\nu\left(r_{t}\right)} \mathcal{E}\left[\nu\left(r_{t}\right)\right]\right]\right. \\
& \left.+\frac{1}{n_{r}}\left[1+\frac{\sqrt{\pi}}{\nu\left(r_{r}\right)} \mathcal{E}\left[\nu\left(r_{r}\right)\right]\right]\right) .
\end{aligned}
$$

From the numerical simulations, $\sigma^{2}$, as well as its upper and lower bounds, decreases monotonically with $n_{t}$ and $n_{r}$ (monotonicity property), and increases with $\left|r_{t}\right|$ and/or $\left|r_{r}\right|$. As the result, similarly to the exponential model, the outage capacity decreases with correlation at outage probabilities less than 0.5.

To demonstrate the effect of correlation structure (the rate of correlation decay with distance $|m-k|$ ) on the outage capacity, Fig. 5 shows $n^{-2}\|\mathbf{R}\|^{2}$ versus $|r|$ for $n=100$ when $\mathbf{R}$ is given by the exponential and QE models. $n^{-2}\|\mathbf{R}\|^{2}$ is numerically evaluated for both models and is shown together with the approximation in (22) (for the exponential model) and the bounds in (26) (for the QE model). For low correlations, $|r|<0.4$, the behavior of $n^{-2}\|\mathbf{R}\|^{2}$ in both models is similar. For $|r| \geq 0.4, n^{-2}\|\mathbf{R}\|^{2}$ in the exponential model increases more rapidly with $|r|$ as compared to the QE model, i.e., the more rapidly the correlation decays with distance, as in the QE model, the higher $r$ can be tolerated without significant loss in capacity. Thus, the effect of correlation on outage capacity is characterized not just by the correlation

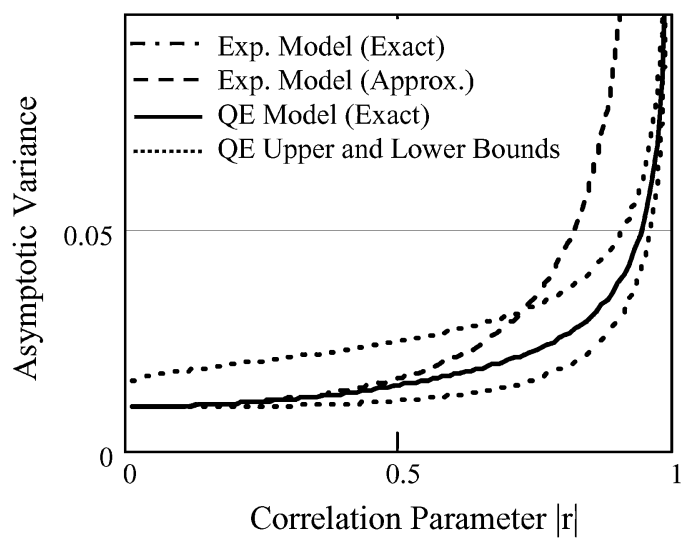

Fig. 5. $n^{-2}\|\mathbf{R}\|^{2}$ in exponential and $\mathrm{QE}$ models versus correlation parameter. The variance of outage capacity distribution follows the same tendency [see (17)].

between adjacent antennas, but also by its rate of decay with distance $|m-k|$, which is accounted for in Definition 1 .

The uniform correlation model [28] can also be considered. However, unlike the exponential and QE, this model does not satisfies the conditions of Theorem $3\left(\lim _{n \rightarrow \infty} n^{-2}\|\mathbf{R}\|^{2} \neq 0\right)$, so it impossible to say based on Theorem 3 whether the outage capacity of a keyhole channel with uniform correlation is asymptotically Gaussian as $n_{t}, n_{r} \rightarrow \infty$.

\section{Convergence Rate and Numerical Results}

From the practical point of view, the asymptotic analysis above is important as an approximation to real channels with a finite number of antennas. From the proof of Theorem 3 (see Appendix C), the outage capacity converges to the Gaussian distribution with the same rate as $n_{t}^{-1}\left\|\mathbf{R}_{t}\right\|$ and $n_{r}^{-1}\left\|\mathbf{R}_{r}\right\|$ go to zero, which is $1 / \sqrt{n}$ ( $n$ is either $n_{t}$ or $n_{r}$ ) for the exponential and QE correlation models [see (22) and (26)]. Extensive numerical simulations were used to assess the accuracy of the asymptotic approximation. Some of the results are shown in Fig. 6 , where the exact capacity distributions of $2 \times 2,3 \times 3$, and $5 \times 5$ keyhole channels with exponential correlation at both Tx and Rx ends are compared to the corresponding Gaussian approximations. Clearly, the difference between the Gaussian approximation and the exact distribution is negligible for most practical purposes. Usually, for finite $n_{t}$ and $n_{r}$, the asymptotic distribution overestimates the exact one. When the approximation (22) and the upper bound in (26) are used in place of the true $\sigma^{2}$, the exact distribution, in some cases, follows closely the asymptotic one already for two antennas at each end. However, we were not able to find a compact general rule indicating the accuracy of the approximation for given parameters.

The asymptotic normality of outage capacity of keyhole channels with Rayleigh-fading subchannels can be generalized for a wider class of keyhole channels.

Theorem 4: Let $C$ be the instantaneous capacity of the correlated keyhole channel where $\mathbf{h}_{t} \propto \mathbf{R}_{t}^{1 / 2} \mathbf{g}_{t}$ and $\mathbf{h}_{r} \propto \mathbf{R}_{r}^{1 / 2} \mathbf{g}_{r}, \propto$ denotes identical distribution, $\mathbf{g}_{t}$ and $\mathbf{g}_{r}$ are zero mean complex random vectors with independent entries (not necessarily complex Gaussian or identically distributed). When both $n_{t}$ and $n_{r}$ tend to infinity, the distribution of $C$ is asymptotically normal if:

i) $m_{2+\delta}(k)<\infty$ and $m_{2}(k)>0$ for all $k$ and some $\delta>0$, where $m_{\delta}(k)=E\left\{\left(\left|g_{k}\right|^{2}-E\left\{\left|g_{k}\right|^{2}\right\}^{\delta}\right\}\right.$ is the central moment of $\left|g_{k}\right|^{2}$ of order $\delta$, and $g_{k}$ is the $k$ th entry of either $\mathbf{g}_{t}$ or $\mathbf{g}_{r}$.

ii) Both $\mathbf{R}_{t}$ and $\mathbf{R}_{r}$ satisfy a Lyapunov-type condition

$$
\lim _{n_{t} \rightarrow \infty} \frac{\|\boldsymbol{\lambda}\|_{2+\delta}}{\|\boldsymbol{\lambda}\|_{2}}=0
$$




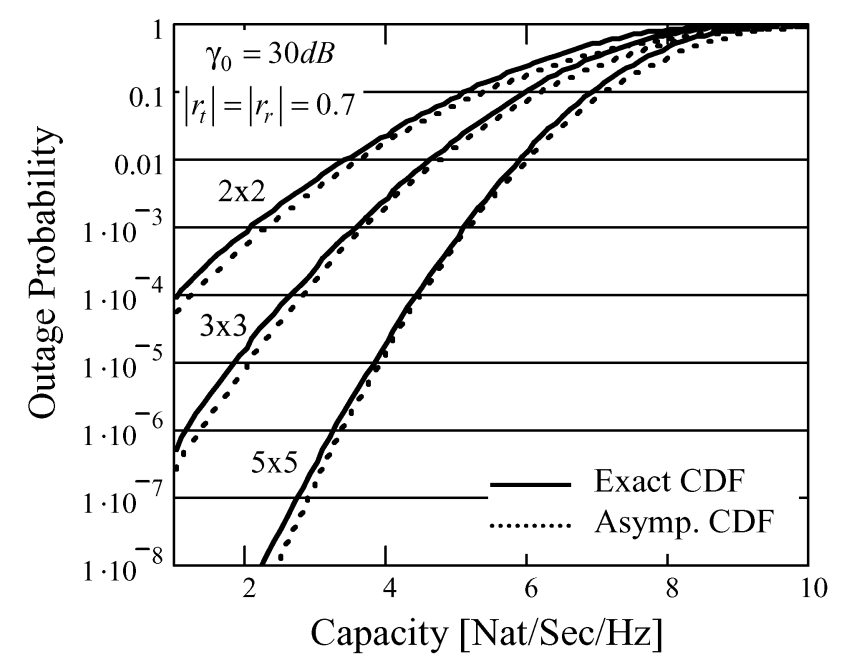

Fig. 6. Keyhole channel exact outage capacity and its asymptotic approximation.

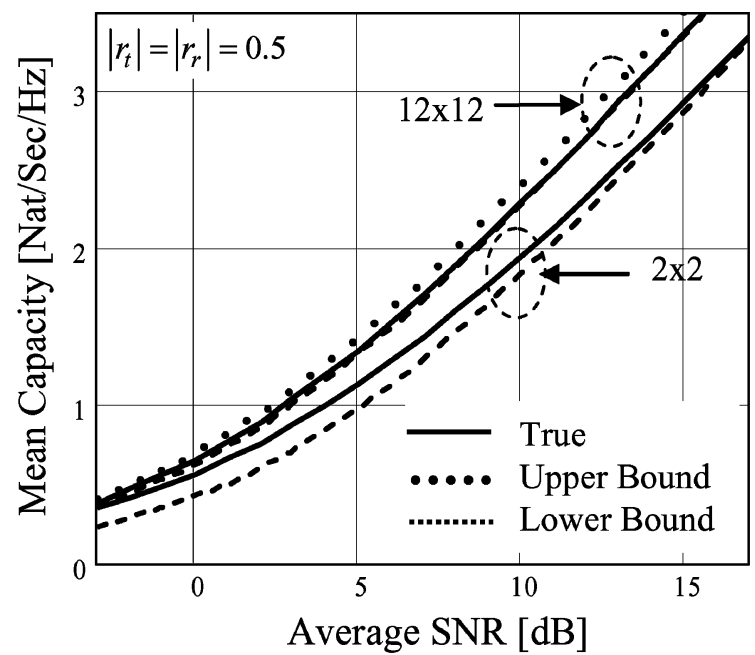

Fig. 7. Mean capacity of correlated keyhole channels versus SNR.

where $\|\boldsymbol{\lambda}\|_{m}=\left(\sum_{i=1}^{n}\left(\lambda_{i}\right)^{m}\right)^{1 / m}$ is the $L_{m}$ norm of the eigenvalues.

Proof: See Appendix E.

Lyapunov-type condition (28) is also important for asymptotic analysis of Rayleigh-fading channels [17]. Its detailed theoretical analysis and some practical implications are presented in [27]. Even though the condition (28) is in a closed form, its usefulness for practical computations is rather limited due to two reasons: 1) The eigenvalues are known in a closed form only for some simple matrices. Consequently, the above condition can be evaluated analytically only in such cases. 2) Numerical evaluation of this condition is also difficult, since the numerical complexity (number of operations, inaccuracy, etc.) of the eigenvalue problem increases rapidly with $n$, so that $n \rightarrow \infty$ is problematic if possible at all. The following Corollary gives a condition that is easier to evaluate.

Corollary 4.1: If both $\mathbf{R}_{t}$ and $\mathbf{R}_{r}$ have Toeplitz structure, i.e., the $k-m$ th element of $\mathbf{R}$ (either $\mathbf{R}_{t}$ or $\mathbf{R}_{r}$ ) is $R_{k m}=R_{k-m}$, then (28) is equivalent to

$$
\lim _{n \rightarrow \infty} n^{-1}\|\mathbf{R}\|^{2}<\infty
$$

for both $\mathbf{R}_{t}$ and $\mathbf{R}_{r}$.
Proof: See Appendix E.

If $\lim _{n \rightarrow \infty} n^{-1}\|\mathbf{R}\|^{2}<\infty$, then $\lim _{n \rightarrow \infty} n^{-2}\|\mathbf{R}\|^{2}=0$, and there is an analogy between the conditions of Theorem 3 and Corollary 4.1 , as both require the measure of correlation $n^{-1}\|\mathbf{R}\|$ to vanish asymptotically. Theorem 3, however, does not require Toeplitz structure of $\mathbf{R}_{t}$ and $\mathbf{R}_{r}$ but is restricted to complex Gaussian $\mathbf{h}_{t}$ and $\mathbf{h}_{r}$. Since Corollary 4.1 applies to a wider class of $\mathbf{h}_{t}$ and $\mathbf{h}_{r}$, it indicates that the measure of correlation proposed in Definition 1 has a higher degree of universality than just for MIMO channels defined through complex Gaussian random vectors.

\section{BOUNDS ON THE MEAN CAPACITY}

Even though the outage capacity is the relevant performance measure for nonergodic fading channels, the mean capacity is also important as it gives an upper limit on error-free information rate supported by ergodic channels. The exact expression for the mean capacity $\bar{C}$ of a correlated keyhole channel is rather complicated and involves Meijer $G$-functions [12]. However, using (3) and Jensen inequality it is straightforward to show that the upper bound on $\bar{C}$ of correlated keyhole channels is

$$
\bar{C} \leq \ln \left(1+\gamma_{0}\right) .
$$

This bound does not depend on correlation and is identical to that proposed by Shin and Lee [12] for the uncorrelated channels. Cui and Feng [14] have proposed a tight lower bound

$$
\bar{C} \geq \ln \left(1+\frac{\gamma_{0}}{n_{t} n_{r}} \exp \left[\Theta\left(\mathbf{R}_{t}\right)+\Theta\left(\mathbf{R}_{r}\right)\right]\right)
$$

where, based on (15) in [14], if $\mathbf{R}$, either $\mathbf{R}_{t}$ or $\mathbf{R}_{r}$, is nonsingular and has distinct eigenvalues,

$$
\Theta(\mathbf{R})=-\gamma_{e}+\sum_{k=1}^{n} A_{k} \ln \left(\lambda_{k}\right)
$$

(for a proof see Appendix F). From Theorem 3, the upper bound (30) is asymptotically tight with respect to the number of antennas at both the Tx and Rx ends, in addition, in Appendix F, we show the following.

i) The lower bound (31) is easily obtained using the instantaneous SNR distribution given by Theorem 1 (this indirectly validates (5), (6)).

ii) While the upper bound (30) is tight as $\gamma_{0} \rightarrow 0$, the lower bound (31) is tight as $\gamma_{0} \rightarrow \infty$ (the last statement has been proven in [14] for noncorrelated channels; we extend this results for correlated channels). To illustrate i) and ii), Fig. 7 shows the mean capacity and its bounds of $2 \times 2$ and $12 \times 12$ keyhole channels versus $\gamma_{0} . \bar{C}$ was numerically evaluated using the instantaneous SNR pdf in (5). The exponential correlation model with the same correlation parameter was used at both the Tx and Rx ends. Clearly, the lower bound is tight (for $12 \times 12$ channel, it is practically indistinguishable from the true mean capacity), the upper bound becomes tight for both $2 \times 2$ and $12 \times 12$ at low SNR.

\section{A. Impact of Correlation}

Unlike the outage capacity, which reduces significantly with correlation, the mean capacity of keyhole channels is almost independent of correlation (even very high one), as Fig. 8 demonstrates. An intuitive explanation of this is that high correlation reduces the effective rank of a full-rank channel; for instance, the rank of a fully correlated $(|r|=1)$ Rayleigh-fading channel is one regardless of the number of antennas. As the result, the mean capacity, in this case, reduces dramatically with correlation [18], primarily due to loss in the effective rank 


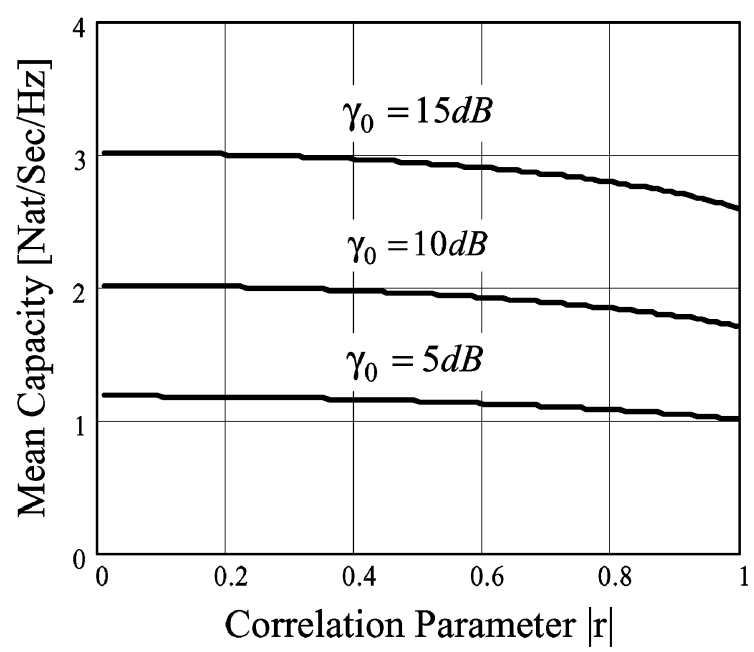

Fig. 8. Mean capacity of $2 \times 2$ keyhole channel with exponential correlation versus correlation parameter $|r|=\left|r_{t}\right|=\left|r_{r}\right|$.

at high correlation. Unlike full-rank channels, the rank of a keyhole channel is already one, regardless of correlation, and hence nor further loss in rank is possible, so that the mean capacity is not affected much.

It also follows from the comparison between the mean capacity of various keyhole channels and the upper bound in (30) that the discrepancy between the two does not exceed $30 \%$ in the worst case. Thus, the upper bound can be used as a simple rough approximation for the mean capacity regardless of correlation.

\section{Achievability of the Keyhole Channel CAPACITy}

It is always intriguing and important to find a system architecture that achieves the capacity. While Alamouti scheme [22] (with appropriate temporal coding) is known to achieve the capacity of channels of $n_{t}=1$ or $n_{r}=1$, it is strictly suboptimal for $n_{t}=2$ rank- 2 channels. However, in keyhole channels, Alamouti scheme achieves the full capacity with either $n_{t}=1$ or $n_{t}=2$ and any $n_{r}$. Theorem 5 below formalizes this result.

Theorem 5: Let $C$ be the instantaneous capacity (2) of a MIMO channel (not necessarily keyhole) with $n_{t}=1$ or $n_{t}=2$ and any $n_{r}$, and $C_{A}$ is the capacity when Alamouti scheme is deployed.

i) In arbitrary channel, $C=C_{A}$ if rank $=1$ (which includes $n_{t}=1$ case), and $C>C_{A}$ if rank $=2$.

ii) In a keyhole channel, $C=C_{A}$.

Proof: From (2),

$$
\begin{aligned}
C & =\ln \operatorname{det}\left(\mathbf{I}+\frac{\gamma_{0}}{n_{t} n_{r}} \mathbf{H} \mathbf{H}^{H}\right) \\
& \geq \ln \left(1+\frac{\gamma_{0}}{n_{t} n_{r}}\|\mathbf{H}\|^{2}\right)=C_{A}
\end{aligned}
$$

where the equality is achieved for rank-1 channels only.

Q.E.D.

Note that Theorem 5 holds for all MIMO channels irrespectively of the underlying fading distribution.

\section{THE IMPACT OF PERFECT TX CSI}

In general, finding the channel capacity when the perfect CSI is available at both Tx and Rx ends is associated with significant mathematical complexity. However, as indicated by the following theorem, in the keyhole channels the effect of Tx CSI is a fixed SNR gain, and therefore does not induce additional complexity into the analysis.
Theorem 6: Consider a keyhole channel with the channel matrix $\mathbf{H}$ given by (1). Under the transmitted power constrain $P_{T} \leq n_{t}$, the perfect Tx CSI at Tx is equivalent to $n_{t}$-fold SNR gain, i.e., the instantaneous channel capacity $C_{\mathrm{CSI}}$ in this case is

$$
C_{\mathrm{CSI}}\left(\gamma_{0}\right)=C\left(n_{t} \gamma_{0}\right)
$$

where $C$ is given by (3). The optimal input covariance matrix that achieves the capacity in (34) is

$$
\mathbf{Q}=\frac{\mathbf{h}_{t} \mathbf{h}_{t}^{H}}{\left\|\mathbf{h}_{t}\right\|^{2}} n_{t}
$$

i.e., the optimal transmission strategy is the beamforming to the keyhole.

Proof: From [1], [35], the instantaneous capacity of the keyhole channel with full CSI at both Tx and Rx ends is given by

$$
\begin{aligned}
C & =\max _{\operatorname{tr}\{\mathbf{Q}\} \leq n_{t}} \ln \operatorname{det}\left(\mathbf{I}+\frac{\gamma_{0}}{n_{t} n_{r}} \mathbf{H} \mathbf{Q} \mathbf{H}^{H}\right) \\
& =\max _{\operatorname{tr}\{\mathbf{Q}\} \leq n_{t}} \ln \operatorname{det}\left(\mathbf{I}+\frac{\gamma_{0}}{n_{t} n_{r}} \mathbf{h}_{r} \mathbf{h}_{t}^{H} \mathbf{Q} \mathbf{h}_{t} \mathbf{h}_{r}^{H}\right)
\end{aligned}
$$

where the second equality is due to (1). Note that $\max _{\operatorname{tr}\{\mathbf{Q}\} \leq n_{t}} \mathbf{h}_{t}^{H} \mathbf{Q} \mathbf{h}_{t}=n_{t}\left\|\mathbf{h}_{t}\right\|^{2}$. The maximum is achieved when $\mathbf{Q}$ has a single nonzero eigenvalue $\lambda_{\max }=n_{t}$, with the corresponding eigenvector $\mathbf{h}_{t} /\left\|\mathbf{h}_{t}\right\|$, i.e., $\mathbf{Q}$ is as in (35). Substituting (35) in (36), one obtains (34).

Since the fixed SNR gain does not change the channel statistics, due Theorem 5, the results obtained above for the keyhole channel with no Tx CSI, including mean and outage capacities, hold true for the channels with the perfect CSI at both ends as well, with the correspondingly adjusted SNR.

\section{OUtAGE CAPACITY AND SyMbol ERROR RATE}

Consider an application of the outage capacity distribution to estimating symbol error rate (SER). If an $M$-ary modulation is used to transmit digital data over a pass-band channel, the maximum rate in natural units per unit bandwidth, which satisfies the zero ISI Nyquist criterion, is $R=\ln (M)$. If $C<R$, the channel is in outage and all the received blocks of symbols are in error with high probability. Assuming that the outage events are the dominant contributor to the block error rate (BLER), it can be estimated via the outage probability

$$
P_{e}(M) \approx \operatorname{Pr}\{C<R\}=F_{C}(\ln (M))
$$

where $F_{C}(x)$ is given by (4). In general, the BLER upper bounds the SER [33]. When the coherence time of the channel significantly exceeds the symbol duration (i.e., long bursts of errors during outage events), the two are close and (37) can serve as an estimate of the SER as well. Table I compares $P_{e}(M)$ in (37) using the exact $F_{\alpha}(x)$ in (6) and the SER of 8-PSK and 16-QAM with Alamouti scheme in the $2 \times 2$ uncorrelated keyhole channel given in [13]. As expected, (37) indeed upper-bounds the SER and is of the same order of magnitude. Thus, the outage probability provides a simple estimation of the SER, which captures the effect of modulation level $M$, without detailed and complicated analysis usually encountered in such problems. Furthermore, for large systems the asymptotic capacity distribution can be used in (37) for this purpose, simplifying the estimation even further.

\section{Multikeyhole Channel}

The ideal keyhole channel is not often encountered in practice [10], [11], since the assumption of a single nonzero eigenmode is only a rough approximation for most propagation scenarios. More often, the 
TABLE I

COMPARISON OF BLER AND SER. FOR CONSISTENCY WITH [13], $\gamma_{0}$ DENOTES THE AVERAGE SNR PER RX ANTENNA

\begin{tabular}{c|c|c|c|c}
\hline \hline \multirow{2}{*}{} & \multicolumn{2}{|c|}{8 -PSK } & \multicolumn{2}{c}{ 16-QAM } \\
\cline { 2 - 5 } & $\gamma_{0}=$ & $\gamma_{0}=$ & $\gamma_{0}=$ & $\gamma_{0}=$ \\
& $10 d B$ & $20 d B$ & $10 d B$ & $20 d B$ \\
\hline$P_{e}(M)$ & $3 \cdot 10^{-1}$ & $1 \cdot 10^{-2}$ & $6 \cdot 10^{-1}$ & $5 \cdot 10^{-2}$ \\
SER & $2 \cdot 10^{-1}$ & $8 \cdot 10^{-3}$ & $2 \cdot 10^{-1}$ & $2 \cdot 10^{-2}$ \\
\hline
\end{tabular}

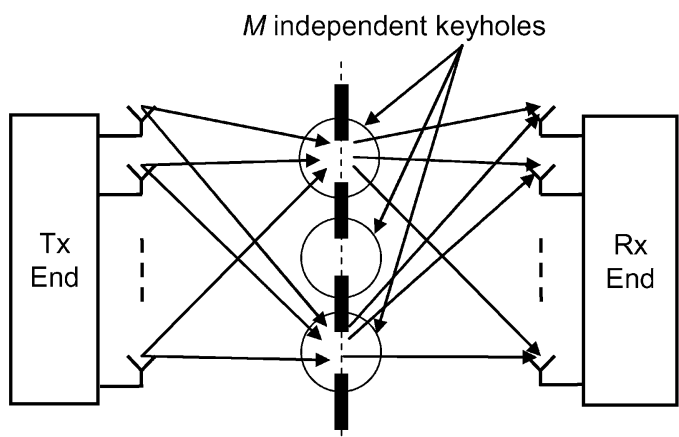

Fig. 9. Multikeyhole MIMO channel.

channels may be comprised of multiple keyholes (see Fig. 9), the "multikeyhole channels." Following (1) and assuming that contribution of the Tx-Rx paths propagating via more than one keyhole is negligible, the transfer matrix of the multikeyhole channel can be represented as:

$$
\mathbf{H}=\sum_{k=1}^{M} a_{k} \mathbf{h}_{\mathrm{rk}} \mathbf{h}_{\mathrm{tk}}^{H}=\mathbf{H}_{r} \mathbf{A} \mathbf{H}_{t}^{H}
$$

where $M$ is a number of keyholes, $a_{k}$ is the complex gain of the $k$ th keyhole, $\mathbf{h}_{t k}\left[n_{t} \times 1\right]$ and $\mathbf{h}_{r k}\left[n_{r} \times 1\right]$ are random vectors representing the complex gains from the transmit antennas to the $k$ th keyhole and from the $k$ th keyhole to the receive antennas, respectively. $\mathbf{H}_{t}=\left[\mathbf{h}_{t 1} \cdots \mathbf{h}_{t M}\right], \mathbf{H}_{r}=\left[\mathbf{h}_{r 1} \cdots \mathbf{h}_{r M}\right]$ are $\left[n_{t} \times M\right]$ and $\left[n_{r} \times M\right]$ matrices, respectively, and $\mathbf{A}$ is a $[M \times M]$ diagonal matrix with elements $\mathbf{A}_{k k}=a_{k}, k=1 \cdots M$. Assume that for every $k, \mathbf{h}_{t k}$ and $\mathbf{h}_{r k}$ are mutually independent complex circular symmetric Gaussian vectors with zero means and corresponding correlation matrices $\mathbf{R}_{t k}=E\left\{\mathbf{h}_{t k} \mathbf{h}_{t k}^{H}\right\}$ and $\mathbf{R}_{r k}=E\left\{\mathbf{h}_{r k} \mathbf{h}_{r k}^{H}\right\}$. Assume also, that the keyholes are independent of each other, i.e., $E\left\{\mathbf{h}_{t k} \mathbf{h}_{t M}^{H}\right\}=E\left\{\mathbf{h}_{r k} \mathbf{h}_{r M}^{H}\right\}=\mathbf{0}$ for any $k \neq m$. For comparison purposes, $\mathbf{H}$ is normalized so that $E\left\{\|\mathbf{H}\|^{2}\right\}=n_{t} n_{r}$ and for every $k, n_{t}^{-1} E\left\{\left\|\mathbf{h}_{t k}\right\|^{2}\right\}=n_{r}^{-1} E\left\{\left\|\mathbf{h}_{r k}\right\|^{2}\right\}=1$, which implies

$$
\sum_{k=1}^{M}\left|a_{k}\right|^{2}=1
$$

i.e., we compare between the channels when the average SNR is constant regardless the number of keyholes.

By substituting (38) in (2), it is straightforward to show that the instantaneous capacity of a frequency-flat quasi-static multikeyhole MIMO channel in natural units with CSI available at Rx end only is given by

$$
C=\ln \operatorname{det}\left(\mathbf{I}+\gamma_{0} \mathbf{B}_{r} \mathbf{A B}_{t} \mathbf{A}^{H}\right)
$$

where $\mathbf{B}_{t}=\mathbf{H}_{t}^{H} \mathbf{H}_{t} / n_{t}$ and $\mathbf{B}_{r}=\mathbf{H}_{r}^{H} \mathbf{H}_{r} / n_{r}$. From (40), the distribution of a full-rank multikeyhole channel with $M \geq \min \left\{n_{t}, n_{r}\right\}$ is different from the full-rank Rayleigh channel.
Theorem 7: Let $C$ be the instantaneous capacity of a multikeyhole channel defined above. If $\lim _{n_{t} \rightarrow \infty} n_{t}^{-1}\left\|\mathbf{h}_{t k}\right\|^{2}<$ $\infty, \lim _{n_{r} \rightarrow \infty} n_{r}^{-1}\left\|\mathbf{h}_{r k}\right\|^{2}<\infty$ and $\lim _{n_{t} \rightarrow \infty} n_{t}^{-2} \operatorname{tr}\left[\mathbf{R}_{t k}^{H} \mathbf{R}_{t M}\right]=$ $0, \lim _{n \rightarrow \infty} n_{r}^{-2} \operatorname{tr}\left[\mathbf{R}_{r k}^{H} \mathbf{R}_{r M}\right]=0$ for every $k, m=1 \cdots M$, then as both $n_{t}, n_{r} \rightarrow \infty$,

$$
C \stackrel{p}{\rightarrow} \sum_{k=1}^{M} \ln \left(1+\frac{\left|a_{k}\right|^{2} \gamma_{0}}{n_{t} n_{r}}\left\|\mathbf{h}_{t k}\right\|^{2}\left\|\mathbf{h}_{r k}\right\|^{2}\right) .
$$

Proof: See Appendix F.

From Theorem 7, the asymptotic instantaneous capacity of a multikeyhole channel is the sum of the capacities of $M$ single keyhole channels, i.e., the subchannels supported by different keyholes are essentially decoupled of each other.

Corollary 6.1: Under the conditions of Theorem 7, the instantaneous capacity of the multikeyhole channel is asymptotically Gaussian with the mean $\mu$ and variance $\sigma^{2}$ as follows:

$$
\begin{aligned}
\mu= & \sum_{k=1}^{M} \ln \left(1+\left|a_{k}\right|^{2} \gamma_{0}\right) \\
\sigma^{2}= & \left(\frac{\gamma_{0}}{1+\gamma_{0}}\right)^{2}\left(\frac{1}{n_{t}^{2}} \sum_{k=1}^{M}\left\|\mathbf{R}_{t k}\right\|^{2}\right. \\
& \left.+\frac{1}{n_{r}^{2}} \sum_{k=1}^{M}\left\|\mathbf{R}_{r k}\right\|^{2}\right) .
\end{aligned}
$$

Proof: Under the conditions of Theorem 7, $C$ in (41) is a sum of independent random variables, which, from Theorem $3,{ }^{4}$ are asymptotically Gaussian. Therefore, $C$ is also asymptotically Gaussian with the mean and variance given by (42).

Using Jensen inequality and the normalization in (39), it is straightforward to show that

$$
\mu \leq M \ln \left(1+\gamma_{0} / M\right)
$$

with equality if $\left|a_{k}\right|=\sqrt{1 / M}, k=1 \cdots M$, i.e., if the gains of all the keyholes are same. Since $M \ln \left(1+\gamma_{0} / M\right)$ increases monotonically with $M$, the channel with more equal-gain keyholes has higher mean capacity. However, this is not necessarily true for the outage capacity, since an increase in $M$ increases not only $\mu$ but also $\sigma^{2}$ (see (42)). Thus, the outage capacity for some outage probabilities may increase, while for others it may decrease. To demonstrate this, consider a marginal case where the channel has $M$ equal-gain keyholes and $M$ is large. From (43), $\lim _{M \rightarrow \infty} \mu=\gamma_{0}$, i.e., asymptotically does not depend on the number of keyholes. In contrast, $\sigma^{2}$ increases with $M$. Thus, from the analysis given in Section IV for two Gaussian cdfs with the same mean and different variances, we conclude that an increase in $M$ decreases the outage capacity of such a multikeyhole channel at outage probabilities less than 0.5 and increases it at outage probabilities greater than 0.5 (we stress that this conclusion holds true under normalization (39) and may change if a different normalization is adopted).

Similarly to (17), $\sigma^{2}$ in (42) is a sum of correlation measures over all keyholes at the Tx and Rx ends. Based on Definition 1 and the analysis presented above, a decrease in correlation and/or an increase in, decrease $\sigma^{2}$ (assuming that for every $k, n^{-1}\left\|\mathbf{R}_{k}\right\|$ decreases monotonically with $n$, where $\mathbf{R}_{k}$ is either $\mathbf{R}_{t k}$ or $\mathbf{R}_{r k}$ ). Therefore, the asymptotic outage capacity increases at outage probabilities less than 0.5 and decreases at outage probabilities greater than 0.5 .

${ }^{4}$ Note that if the conditions of Theorem 5 are fulfilled, then the conditions of Theorem 3 are also satisfied. 


\section{CONCLUSION}

A profound reason to study keyhole channels is not only in practical applications (i.e., as a model of physical propagation channels, including relay amplify-and-forward channels), but also due to the unique position of these channels as a model of the worst case MIMO propagation scenario (i.e., rank-one MIMO channels). The investigation of the keyhole channels can reveal how well a system performs in channels other than the Rayleigh ones (which were extensively studied and are well understood by now), and how much the results established for the Rayleigh channels apply elsewhere (i.e., robustness). The present study shows that, despite the degenerate nature of keyhole channels, their outage capacity is, similarly to full-rank Rayleigh channels, asymptotically Gaussian, when the number of antennas is large, regardless of whether or not the channel state information is available at the Tx end. This conclusion, together with other results on the asymptotic outage capacity of uncorrelated and correlated Rayleigh channels [16], [17] indicates that the Gaussian distribution has a high degree of universality (robustness) in the outage capacity analysis of MIMO channels in general.

\section{APPENDIX A}

\section{PROOF OF THEOREM 1}

Let $\beta$ be either $\beta_{t}=\left\|\mathbf{h}_{t}\right\|^{2}, \beta_{r}=\left\|\mathbf{h}_{r}\right\|^{2}$. Under the adopted assumptions, the distribution of $\beta$ is generalized $\chi^{2}$ with the characteristic function $\Phi_{\beta}(\omega)=\operatorname{det}^{-1}[\mathbf{I}-j \omega \mathbf{R}]$, where $\mathbf{R}$ is either $\mathbf{R}_{t}$ or $\mathbf{R}_{r}$, and $j=\sqrt{-1}$ [29]. When $\mathbf{R}$ is nonsingular and has $n$ distinct eigenvalues $\lambda_{k}, k=1 \cdots n$. The characteristic function (CF) of $\beta$ can be represented as

$$
\Phi_{\beta}(\omega)=\prod_{k=1}^{n}\left(1-j \omega \lambda_{k}\right)^{-1}=\sum_{k=1}^{n} A_{k}\left(1-j \omega \lambda_{k}\right)^{-1}
$$

where $A_{k}$ are the coefficients of the partial fraction decomposition of $\Phi_{\beta}(\omega)$, such that $\sum_{k=1}^{n} A_{k}=1$ [24]. From (A1)

$$
\sum_{k=1}^{n} A_{k} \prod_{\substack{m=1 \\ m \neq k}}^{n}\left(1-j \omega \lambda_{m}\right)=1
$$

Since the equality in (A2) holds for every $\omega$, at $\omega=-j \lambda_{k}^{-1}, k=$ $1 \cdots n(\mathrm{~A} 2)$ is

$$
A_{k} \prod_{\substack{m=1 \\ m \neq k}}^{n}\left(1-\lambda_{m} / \lambda_{k}\right)=1
$$

which proves (7).

Based on (A1), the pdf and the cdf of $\beta$ are given for $x \geq 0$ by

$$
\begin{aligned}
f_{\beta}(x) & =\frac{1}{2 \pi} \int_{-\infty}^{\infty} \Phi_{\beta}(\omega) e^{-j \omega x} d \omega \\
& =\sum_{k=1}^{n} \frac{A_{k}}{\lambda_{k}} \exp \left(-\frac{x}{\lambda_{k}}\right) \\
F_{\beta}(x) & =\int_{0}^{x} f_{\beta}(t) d t=1-\sum_{k=1}^{n} A_{k} \exp \left(-\frac{x}{\lambda_{k}}\right) .
\end{aligned}
$$

Since $\alpha$ is a product of $\beta_{t}, \beta_{r}$, and $\beta_{t}, \beta_{r}$ are assumed to be independent, the pdf $f_{\alpha}(z)$ and the $\operatorname{cdf} F_{\alpha}(z)$ of $\alpha$ are

$$
\begin{aligned}
& f_{\alpha}(z)=\int_{0}^{\infty} f_{\beta_{t}}(z / x) f_{\beta_{r}}(x) d \ln (x) \\
& F_{\alpha}(z)=\int_{0}^{\infty} F_{\beta_{t}}(z / x) f_{\beta_{r}}(x) d x
\end{aligned}
$$

where $F_{\beta_{t}}(x), f_{\beta_{t}}(x)$ and $F_{\beta_{r}}(x), f_{\beta_{r}}(x)$ are the cdf and the pdf of $\beta_{t}$ and $\beta_{r}$, respectively. Thus, by substituting (A4) and (A5) in (A6) and (A7) one obtains (5) and (6).

Q.E.D.

\section{APPENDIX B}

\section{PROOF OF THEOREM 2 AND COROLLARY 2.1}

Lemma $B$ : Let $\beta$ be a generalized $\chi^{2}$ random variable with the $\mathrm{CF}$ $\Phi_{\beta}(\omega)=\operatorname{det}^{-1}\left[\mathbf{I}-j \omega \mathbf{R}\right.$ ] [29]. If $\lim _{n \rightarrow \infty} n^{-1} \operatorname{tr}\{\mathbf{R}\}<\infty$ and $\lim _{n \rightarrow \infty} n^{-2}\|\mathbf{R}\|^{2}=0$, then, as $n \rightarrow \infty$, the distribution of $n^{-1} \beta$ is Gaussian with the mean $\mu=n^{-1} \operatorname{tr}\{\mathbf{R}\}$ and the variance $\sigma^{2}=$ $n^{-2}\|\mathbf{R}\|^{2}$.

Proof: Since $\beta$ is a generalized $\chi^{2}$ random variable with $\mathrm{CF}$ $\Phi_{\beta}(\omega)=\operatorname{det}^{-1}[\mathbf{I}-j \omega \mathbf{R}]$, the characteristic function of $n^{-1} \beta$ is $\Phi(\omega)=\prod_{k=1}^{n}\left(1-j \omega \lambda_{k} / n\right)^{-1}$, where $\lambda_{k}$ are the eigenvalues of $\mathbf{R}$. Or equivalently $\ln (\Phi(\omega))=-\sum_{k=1}^{n} \ln \left(1-j \omega \lambda_{k} / n\right)$. Assume that $n$ is large enough such that for every $\lambda_{k},\left|\omega \lambda_{k} / n\right|<1$, then the expansion of $\ln (\Phi(\omega))$ in Maclaurin series gives

$$
\ln (\Phi(\omega))=\sum_{k=1}^{n} \sum_{m=1}^{\infty} \frac{\left(j \omega \lambda_{k}\right)^{m}}{n^{m} m}
$$

Define $L_{m}=n^{-m} \sum_{k=1}^{n} \lambda_{k}^{m}, m=1,2, \ldots$ By changing the order of summation, (B1) can be rewritten as

$$
\ln (\Phi(\omega))=\ln \left(\Phi_{g}(\omega)\right)+\sum_{m=3}^{\infty}(j \omega)^{m} L_{m} / m
$$

where $\Phi_{g}(\omega)=\exp \left(j \omega L_{1}-\omega^{2} L_{2} / 2\right)$ is the CF of a Gaussian random variable with the mean $\mu=L_{1}$ and the variance $\sigma^{2}=L_{2}$. Note that $L_{1}=n^{-1} \operatorname{tr}(\mathbf{R})$ and $L_{2}=n^{-2}\|\mathbf{R}\|^{2}$. Therefore, the necessary conditions for $\lim _{n \rightarrow \infty} \Phi(\omega)=\Phi_{g}(\omega)$ are

$$
\lim _{n \rightarrow \infty} L_{1}<\infty, \lim _{n \rightarrow \infty} L_{2}<\infty \lim _{n \rightarrow \infty} L_{m} / L_{2}=0 \text { for } m \geq 3
$$

Below we show in two steps that the sufficient condition to ensure $\lim _{n \rightarrow \infty} L_{m} / L_{2}=0$ for $m \geq 3$ is $\lim _{n \rightarrow \infty} L_{2}=0$. Note that $\left|\omega \lambda_{k} / n\right|<1$ follows from $\lim _{n \rightarrow \infty} L_{2}=0$, and hence it holds true under the conditions of Lemma B.

1) If $\lim _{n \rightarrow \infty} L_{2}=0$ then $\lim _{n \rightarrow \infty} L_{2 m} / L_{2}=0$ for $m=2,3, \ldots$

$$
\begin{aligned}
\lim _{n \rightarrow \infty} L_{2 m} / L_{2} & =\lim _{n \rightarrow \infty} L_{2}^{-1} n^{-2 m} \sum_{k=1}^{n} \lambda_{k}^{2 m} \\
& \leq \lim _{n \rightarrow \infty} L_{2}^{-1}\left(n^{-2} \sum_{k=1}^{n} \lambda_{k}^{2}\right)^{m} \\
& =\lim _{n \rightarrow \infty} L_{2}^{m-1}=0 .
\end{aligned}
$$


The inequality is because all $\lambda_{k}$ are nonnegative. Thereby, since $\lim _{n \rightarrow \infty} L_{m} / L_{2} \geq 0$

$$
\lim _{n \rightarrow \infty} L_{2 m} / L_{2}=0, \quad m \geq 2 .
$$

2) Based on (B5) and Cauchy-Schwarz's inequality, for $m \geq 2$

$$
\begin{aligned}
& \lim _{n \rightarrow \infty} L_{m+1} / L_{2} \\
& \quad=\lim _{n \rightarrow \infty} L_{2}^{-1} n^{-(m+1)} \sum_{k=1}^{n} \lambda_{k}^{m+1} \leq \\
& \quad \leq \lim _{n \rightarrow \infty} L_{2}^{-1} \sqrt{n^{-2 m} \sum_{k=1}^{n} \lambda_{k}^{2 m}} \cdot \sqrt{n^{-2} \sum_{k=1}^{n} \lambda_{k}^{2}} \\
& \quad=\lim _{n \rightarrow \infty} L_{2}^{-1} \sqrt{L_{2 m}} \cdot \sqrt{L_{2}} \\
& \quad=\lim _{n \rightarrow \infty} \sqrt{L_{2 m} / L_{2}}=0 .
\end{aligned}
$$

Using the same argument as for (B5)

$$
\lim _{n \rightarrow \infty} L_{m+1} / L_{2}=0, \quad m \geq 2 .
$$

Therefore, if $\lim _{n \rightarrow \infty} n^{-1} \operatorname{tr}(\mathbf{R})<\infty$ and $\lim _{n \rightarrow \infty} n^{-2}\|\mathbf{R}\|^{2}=0, \lim _{n \rightarrow \infty} \Phi(\omega)=\Phi_{g}(\omega)$, i.e., $n^{-1} \beta$ is asymptotically Gaussian in distribution.

Q.E.D.

Corollary B: Under the conditions of Lemma B

$$
n^{-1} \beta \stackrel{p}{\rightarrow} 1 \text { as } n \rightarrow \infty
$$

Proof: From Chebyshev inequality [29], for any $\varepsilon>0$ :

$$
\operatorname{Pr}\left\{\left|n^{-1} \beta-E\left\{n^{-1} \beta\right\}\right| \geq \varepsilon\right\} \leq \operatorname{Var}\left\{n^{-1} \beta\right\} / \varepsilon^{2}
$$

where Var denotes variance. Since under the adopted normalization $E\left\{n^{-1} \beta\right\}=1$ and following Lemma $\mathrm{B} \operatorname{Var}\left\{n^{-1} \beta\right\}=n^{-2}\|\mathbf{R}\|^{2} \rightarrow$ 0 as $n \rightarrow \infty$, using the continuity property of probability measure, one obtains

$$
\operatorname{Pr}\left(\left|\lim _{n \rightarrow \infty} \frac{\beta}{n}-1\right| \geq \varepsilon\right) \leq \lim _{n \rightarrow \infty} \frac{\operatorname{Var}(\beta / n)}{\varepsilon^{2}}=0 .
$$

Q.E.D.

\section{Proof of Theorem 2:}

i) $n_{t} \rightarrow \infty, n_{r}<\infty$ : From Lemma B and Corollary B, $n_{t}^{-1} \beta_{t} \stackrel{p}{\rightarrow}$ 1 as $n_{t} \rightarrow \infty$. Thus, from Slutsky Theorem [[30, Th. 6'a]], $C \stackrel{p}{\rightarrow}$ $\ln \left(1+\gamma_{0} \beta_{r} / n_{r}\right)$ as $n_{t} \rightarrow \infty$. Note that under the adopted assumptions $\ln \left(1+\gamma_{0} \beta_{r} / n_{r}\right)$ is the instantaneous capacity of an $1 \times n_{r}$ Rayleigh fading channel.

ii) $n_{t}<\infty, n_{r} \rightarrow \infty$ : Due to the symmetry, the proof follows the same arguments when the Tx and Rx ends exchanged. Q.E.D.

\section{Proof of Corollary 2.1:}

i) $n_{t} \rightarrow \infty, n_{r}<\infty$ : Let $J=\ln \left(1+\gamma_{0} \beta_{r} / n_{r}\right)$. Since $J$ is a continuous monotonically increing function of $\beta_{r}$, its cdf is $F_{J}(x)=F_{\beta_{r}}\left(n_{r}\left(e^{x}-1\right) / \gamma_{0}\right)$. Thus, from Theorem 2 and using the fact that convergence in probability implies convergence in distribution, as $n_{t} \rightarrow \infty$

$$
F_{C}(x) \rightarrow F_{J}(x)=F_{\beta_{r}}\left(n_{r}\left(e^{x}-1\right) / \gamma_{0}\right) .
$$

If $\mathbf{R}_{r}$ is nonsingular and has distinct eigenvalues $\lambda_{t}^{r}, k=$ $1 \cdots n_{t}, F_{\beta_{r}}(x)$ is given by (A5).

ii) $n_{t}<\infty, n_{r} \rightarrow \infty$ : Due to the symmetry, the proof follows the same arguments when the Tx and Rx ends exchanged. Q.E.D.

\section{APPENDIX C \\ PROOF OF THEOREM 3}

Define a function $f(x, y)=\ln \left(1+\gamma_{0} x \cdot y\right)$. From (3), $C=f\left(n_{t}^{-1} \beta_{t}, n_{r}^{-1} \beta_{r}\right)$. From Lemma $\mathrm{B}$, as $n_{t} \rightarrow \infty$ and $n_{r} \rightarrow \infty, n_{t}^{-1} \beta_{t}$ and $n_{r}^{-1} \beta_{r}$ are asymptotically Gaussian in distribution with the means $E\left(n_{t}^{-1} \beta_{t}\right)=E\left(n_{r}^{-1} \beta_{r}\right)=1$ and the variances $n_{t}^{-2}\left\|\mathbf{R}_{t}\right\|^{2}$ and $n_{r}^{-2}\left\|\mathbf{R}_{r}\right\|^{2}$. Since the derivative of $f(x, y)$ is continuous in the neighborhood of $x=1$ and $y=1$, using Cramer Theorem [31], [30, Th. 7], $C$ is asymptotically Gaussian with the mean

$$
\mu=f(1,1)=\ln \left(1+\gamma_{0}\right)
$$

and the variance

$$
\begin{aligned}
x \sigma^{2}= & {\left[\left.\frac{\partial f(x, y)}{\partial x}\right|_{\substack{x=1 \\
y=1}}\right]^{2} \frac{\left\|\mathbf{R}_{t}\right\|^{2}}{n_{t}^{2}} } \\
& +\left[\left.\frac{\partial f(x, y)}{\partial y}\right|_{\substack{x=1 \\
y=1}}\right]^{2} \frac{\left\|\mathbf{R}_{r}\right\|^{2}}{n_{r}^{2}} \\
= & \left(\frac{\gamma_{0}}{1+\gamma_{0}}\right)^{2}\left(\frac{1}{n_{t}^{2}}\left\|\mathbf{R}_{t}\right\|^{2}+\frac{1}{n_{r}^{2}}\left\|\mathbf{R}_{r}\right\|^{2}\right) .
\end{aligned}
$$

Q.E.D.

\section{APPENDIX D \\ APPROXIMATIONS OF CORRELATION MODELS}

\section{A. Exponential Model}

Consider an $n \times n$ exponential correlation matrix $\mathbf{R}$ whose elements are defined in (21). Then

$$
n^{-1} \operatorname{tr}\{\mathbf{R}\}=1<\infty
$$

For a finite $n$ and $|r| \neq 1,\|\mathbf{R}\|^{2}$ is

$$
\begin{aligned}
\|\mathbf{R}\|^{2} & =\operatorname{tr}\{\mathbf{R R}\}=2 \sum_{k=0}^{n-1} \sum_{m=0}^{n-k-1}|r|^{2 m}-n= \\
& =2 \sum_{k=0}^{n-1} \frac{1-|r|^{2(n-k)}}{1-|r|^{2}}-n \\
& =\frac{2}{1-|r|^{2}}\left[n-\sum_{m=1}^{n}|r|^{2 m}\right]-n \\
& =\frac{n\left(1+|r|^{2}\right)}{1-|r|^{2}}+\frac{2\left(|r|^{2(n+1)}-|r|^{2}\right)}{\left(1-|r|^{2}\right)^{2}} .
\end{aligned}
$$

Therefore as $n \rightarrow \infty$

$$
n^{-2}\|\mathbf{R}\|^{2} \rightarrow \frac{1}{n} \cdot \frac{1+|r|^{2}}{1-|r|^{2}} \rightarrow 0|r|<1 .
$$

Q.E.D.

\section{B. Quadratic Exponential Model}

Consider an $n \times n$ quadratic exponential correlation matrix $\mathbf{R}$ whose elements are defined in (25). Then

$$
n^{-1} \operatorname{tr}\{\mathbf{R}\}=1<\infty
$$


For a finite $n,\|\mathbf{R}\|^{2}$ is

$$
\|\mathbf{R}\|^{2}=\operatorname{tr}\{\mathbf{R R}\}=2 \sum_{k=0}^{n-1} \sum_{m=0}^{n-k-1}|r|^{2 m^{2}}-n
$$

$\|\mathbf{R}\|^{2}$ can be bounded using Cauchy convergence test [24] which is based on the following: Let $a_{k}$ be a decreasing sequence of $k \in Z$ (a set of integer numbers) and $a(x)$ be a monotonically decreasing function such that $a(x)=a_{k}$ at $x=k$, then

$$
\int_{0}^{n+1} a(x) d x \leq \sum_{k=0}^{n} a_{k} \leq \int_{-1}^{n} a(x) d x .
$$

Let $b_{k}$ be an increasing sequence of $k \in Z$ and $b(x)$ be a monotonically increasing function such that $b(x)=b_{k}$ at $x=k$, then:

$$
\int_{-1}^{n} b(x) d x \leq \sum_{k=0}^{n} b_{k} \leq \int_{0}^{n+1} b(x) d x .
$$

1) Upper bound on $n^{-2}\|\mathbf{R}\|^{2}$ for $n \rightarrow \infty$ : Let $a_{k}=$ $\sum_{m=0}^{n-k-1}|r|^{2 m^{2}}$. Since for $|r|<1 a_{k}$ is a sum of the decreasing sequence $|r|^{2 m^{2}}$, we use (D6) to obtain

$$
\begin{aligned}
a_{k} & =1+\sum_{m=1}^{n-k-1}|r|^{2 m^{2}} \leq 1+\int_{0}^{n-k-1}|r|^{2 x^{2}} d x \\
& =1+\frac{\sqrt{\pi}}{2} \cdot \frac{\mathcal{E}_{1}[(n-k-1) \nu(r)]}{\nu(r)}
\end{aligned}
$$

where $\nu(r)=\sqrt{-2 \ln |r|}, \mathcal{E}_{1}(x)=\operatorname{erf}(x)$ is the error function [24]. From (D5)

$$
\begin{aligned}
\|\mathbf{R}\|^{2}= & 2 \sum_{k=0}^{n-1} a_{k}-n \leq n \\
& +\frac{\sqrt{\pi}}{\nu(r)} \sum_{k=0}^{n-1} \mathcal{E}_{1}[(n-k-1) \nu(r)] \\
= & n+\frac{\sqrt{\pi}}{\nu(r)} \sum_{m=0}^{n-1} \mathcal{E}_{1}[m \cdot \nu(r)] \leq n \\
& +\frac{\sqrt{\pi}}{\nu(r)} \int_{0}^{n} \mathcal{E}_{1}[x \cdot \nu(r)] d x \\
= & n+\frac{n \sqrt{\pi}}{\nu(r)} \mathcal{E}_{1}[n \cdot \nu(r)] \\
& +\frac{1-\exp \left[-(n \cdot \nu(r))^{2}\right]}{-\nu^{2}(r)} .
\end{aligned}
$$

The second inequality is due to (D7), since $\mathcal{E}_{1}[m \cdot \nu(r)]$ is an increasing sequence of $m$. Thereby, as $n \rightarrow \infty$

$$
n^{-2}\|\mathbf{R}\|^{2} \leq \frac{1}{n} \cdot\left[1+\frac{\sqrt{\pi}}{\nu(r)}\right] \rightarrow 0 .
$$

2) Lower bound on $n^{-2}\|\mathbf{R}\|^{2}$ for $n \rightarrow \infty$ : From (D6) for $|r|<1$

$$
\begin{aligned}
a_{k} & =1+\sum_{m=1}^{n-k-1}|r|^{2 m^{2}} \geq 1+\int_{1}^{n-k}|r|^{2 x^{2}} d x \\
& =1+\frac{\sqrt{\pi}}{2 \nu(r)}\left(\mathcal{E}_{1}[(n-k) \nu(r)]-\mathcal{E}_{1}[\nu(r)]\right)
\end{aligned}
$$

Then following (D5)

$$
\begin{gathered}
\|\mathbf{R}\|^{2}=2 \sum_{k=0}^{n-1} a_{k}-n \geq n \\
+\frac{\sqrt{\pi}}{\nu(r)} \sum_{k=0}^{n-1} \mathcal{E}_{1}[(n-k) \nu(r)] \\
-\frac{n \sqrt{\pi}}{\nu(r)} \mathcal{E}_{1}[\nu(r)]=n+\frac{\sqrt{\pi}}{\nu(r)} \sum_{m=1}^{n} \mathcal{E}_{1}[m \cdot \nu(r)] \\
-\frac{n \sqrt{\pi}}{\nu(r)} \mathcal{E}_{1}[\nu(r)] \geq n+\frac{\sqrt{\pi}}{\nu(r)} \int_{0}^{n} \mathcal{E}_{1}[x \cdot \nu(r)] d x \\
-\frac{n \sqrt{\pi}}{\nu(r)} \mathcal{E}_{1}[\nu(r)]=n+\frac{n \sqrt{\pi}}{\nu(r)}\left(\mathcal{E}_{1}[n \cdot \nu(r)]-\mathcal{E}_{1}[\nu(r)]\right) \\
\quad+\frac{1-\exp \left[-(n \cdot \nu(r))^{2}\right]}{-\nu^{2}(r)} \\
\text { i.e., as } n \rightarrow \infty \quad
\end{gathered}
$$

$$
\frac{1}{n^{2}}\|\mathbf{R}\|^{2} \geq \frac{1}{n}\left[1+\frac{\sqrt{\pi}}{\nu(r)} \mathcal{E}[\nu(r)]\right] \rightarrow 0
$$

where $\mathcal{E}(x)=\operatorname{erfc}(x)$ is the complementary error function [24]

Q.E.D.

\section{APPENDIX E}

\section{ProOF OF ThEOREM 4, COROLLARY 4.1}

Lemma E: Let $\mathbf{h} \propto \mathbf{R}^{1 / 2} \mathbf{g}$, where $\mathbf{g}$ is an $n \times 1$ zero mean complex random vector with independent entries, and $\mathbf{R}$ is an $n \times n$ correlation matrix. As $n \rightarrow \infty, n^{-1}\|\mathbf{h}\|^{2}$ is asymptotically normal in distribution if: $m_{2+\delta}(k)<\infty$ and $m_{2}(k)>0$ for all $k$ and some $\delta>0$, where $m_{\delta}(k)=E\left\{\left(\left|g_{k}\right|^{2}-E\left\{\left|g_{k}\right|^{2}\right\}^{\delta}\right\}\right.$ is the central moment of $\left|g_{k}\right|^{2}$ of order $\delta$, and $g_{k}$ is the $k$ th entry of $\mathbf{g}$, and 2)

$$
Z(\delta)=\lim _{n \rightarrow \infty}\|\boldsymbol{\lambda}\|_{2+\delta} /\|\lambda\|_{2}=0
$$

where $\|\boldsymbol{\lambda}\|_{m}=\left(\sum_{i=1}^{n}\left(\lambda_{i}\right)^{m}\right)^{1 / m}$ is the norm of the eigenvalues of R.

Proof: Under the adopted assumptions

$$
n^{-1}\|\mathbf{h}\|^{2} \propto n^{-1} \sum_{k=1}^{n} \lambda_{k}\left|g_{k}\right|^{2} .
$$

From Lyapunov Theorem [[32, p. 310]] $n^{-1}\|\mathbf{h}\|^{2}$ is asymptotically normal in distribution as $n \rightarrow \infty$, if for some $\delta>0$

$$
\lim _{n \rightarrow \infty} \frac{\left(\sum_{k=1}^{n} \lambda_{k}^{2+\delta} m_{2+\delta}(k)\right)^{1 /(2+\delta)}}{\left(\sum_{k=1}^{n} \lambda_{k}^{2} \cdot m_{2}(k)\right)^{1 / 2}}=0
$$

Let $M=\max _{k}\left\{m_{2+\delta}(k)\right\}<\infty$, and $m=\min _{k}\left\{m_{2}(k)\right\}>0$, then

$$
\begin{aligned}
\lim _{n \rightarrow \infty} & \frac{\left(\sum_{k=1}^{n} \lambda_{k}^{2+\delta} m_{2+\delta}(k)\right)^{1 /(2+\delta)}}{\left(\sum_{k=1}^{n} \lambda_{k}^{2} \cdot m_{2}(k)\right)^{1 / 2}} \\
\leq & \lim _{n \rightarrow \infty} \frac{M^{1 /(2+\delta)}}{m^{1 / 2}} \frac{\left(\sum_{k=1}^{n} \lambda_{k}^{2+\delta}\right)^{1 /(2+\delta)}}{\left(\sum_{k=1}^{n} \lambda_{k}^{2}\right)^{1 / 2}} \\
= & \frac{M^{1 /(2+\delta)}}{m^{1 / 2}} Z(\delta) .
\end{aligned}
$$


Under the conditions of Lemma E, $M^{1 /(2+\delta)} / m^{1 / 2}<\infty$. Thereby, from (E4), if $Z(\delta)=0$, the limit in (E3) holds, i.e., $n^{-1}\|\mathbf{h}\|^{2}$ is asymptotically normal in distribution.

Q.E.D.

Proof of Theorem 4: Define a function $f(x, y)=\ln \left(1+\gamma_{0} x \cdot y\right)$. From (3), $C=f\left(n_{t}^{-1} \beta_{t}, n_{r}^{-1} \beta_{r}\right)$. From Lemma $\mathrm{E}$ and under the adopted normalization, as $n_{t} \rightarrow \infty$ and $n_{r} \rightarrow \infty, n_{t}^{-1} \beta_{t}$ and $n_{r}^{-1} \beta_{r}$ are asymptotically Gaussian in distribution with the means $E\left(n_{t}^{-1} \beta_{t}\right)=E\left(n_{r}^{-1} \beta_{r}\right)=1$. Since $C$ is a smooth function (first-order derivative is continuous) in the neighborhood of $E\left(n_{t}^{-1} \beta_{t}\right)$ and $E\left(n_{r}^{-1} \beta_{r}\right)$, using Cramer Theorem [31], [[30, Th. 7]], $C$ is asymptotically Gaussian

Q.E.D.

Proof of Corollary 4.1: Let $\mathbf{R}$ (either $\mathbf{R}_{t}$ or $\mathbf{R}_{r}$ ) be a Toeplitz correlation matrix with vector eigenvalues $\boldsymbol{\lambda}$. Let $t_{k-m}$ be the $k, m$ element of R. From [27, Th. 1], $Z(\delta)=\lim _{n_{t} \rightarrow \infty}\|\boldsymbol{\lambda}\|_{2+\delta} /\|\boldsymbol{\lambda}\|_{2}=0$ for any $\delta>0$ if

$$
\lim _{n \rightarrow \infty} \sum_{k=-n+1}^{n-1}\left|t_{k}\right|^{2}<\infty
$$

i.e., $\mathbf{R}$ is square-summable. From Szego Theorem [34]

$$
\begin{aligned}
& \lim _{n \rightarrow \infty} \sum_{k=-n+1}^{n-1}\left|t_{k}\right|^{2} \\
& =\lim _{n \rightarrow \infty} n^{-1} \sum_{k=0}^{n-1} \lambda_{k}^{2} \\
& =\lim _{n \rightarrow \infty} n^{-1}\|\mathbf{R}\|^{2}
\end{aligned}
$$

where $\lambda_{k}$ are the eigenvlues of $\mathbf{R}$. Thus, if $\lim _{n \rightarrow \infty} n^{-1}\|\mathbf{R}\|^{2}<\infty$, then $Z(\delta)=0$ for any $\delta>0$.

Q.E.D.

\section{APPENDIX F}

\section{BOUNDS ON MEAN CAPACITY}

From (3) and following the approach proposed in [14]

$$
\begin{aligned}
\bar{C} & =E\left\{\ln \left(1+\frac{\gamma_{0}}{n_{t} n_{r}} \cdot \alpha\right)\right\} \\
& \geq \ln \left(1+\frac{\gamma_{0}}{n_{t} n_{r}} \cdot \exp [E\{\ln \alpha\}]\right) .
\end{aligned}
$$

The inequality is due to the convexity of $\ln (1+\exp [x])$. The generalized moments of $\alpha$ are

$$
\begin{aligned}
E\left\{\alpha^{u}\right\}= & \int_{0}^{\infty} x^{u} d F_{\alpha}(x) \\
= & \Gamma^{2}[u+1] \cdot \sum_{k=1}^{n_{t}} \\
& A_{k}^{t}\left(\lambda_{k}^{t}\right)^{u} \cdot \sum_{m=1}^{n_{r}} A_{m}^{r}\left(\lambda_{m}^{r}\right)^{u}
\end{aligned}
$$

where $F_{\alpha}(x)$ is given by (6), $\Gamma(x)$ is the Gamma function [24], $\lambda_{k}^{t}, \lambda_{m}^{r}$ are the eigenvalues of the correlation matrices $\mathbf{R}_{t}$ and $\mathbf{R}_{r}$, and both $A_{k}^{t}, A_{m}^{r}$ are given by (7) when $\lambda_{k}^{t}, \lambda_{m}^{r}$ are distinct and nonzero. Substituting (F2) in $E\{\ln \alpha\}=\left.(d / d u) E\left\{\alpha^{u}\right\}\right|_{u=0}$ one obtains

$$
E\{\ln \alpha\}=\Theta\left(\mathbf{R}_{t}\right)+\Theta\left(\mathbf{R}_{r}\right)
$$

where $\Theta\left(\mathbf{R}_{t}\right)=-\gamma_{e}+\sum_{k=1}^{n_{t}} A_{k}^{t} \ln \left(\lambda_{k}^{t}\right)$ and $\Theta\left(\mathbf{R}_{t}\right)=-\gamma_{e}+$ $\sum_{m=1}^{n_{r}} A_{m}^{r} \ln \left(\lambda_{m}^{r}\right)$. Substituting (F3) in (F1) gives the lower bound in (31).
Denote the upper and lower bounds in (30) and (F1) by $C_{u}$ and $C_{l}$, respectively. From $(\mathrm{F} 1)$, as $\gamma_{0} \rightarrow \infty, \bar{C} \rightarrow \ln \left(\gamma_{0} /\left(n_{t} n_{r}\right)\right)+E\{\ln \alpha\}$, and so $C_{l} \rightarrow \ln \left(\gamma_{0} /\left(n_{t} n_{r}\right)\right)+E\{\ln \alpha\}$, thus, $C_{l} \rightarrow \bar{C}$ as $\gamma_{0} \rightarrow$ $\infty$.

Q.E.D.

From the expansion of the right hand side of (F1) and (30) in Maclaurin series, as $\gamma_{0} \rightarrow 0, \bar{C} \rightarrow\left(\gamma_{0} /\left(n_{t} n_{r}\right)\right) E\{\alpha\}=\gamma_{0}$, and so $C_{u} \rightarrow \gamma_{0}$, where $E\{\alpha\}=n_{t} n_{r}$ due to the adopted normalization. Therefore $C_{u} \rightarrow \bar{C}$ as $\gamma_{0} \rightarrow 0$

Q.E.D.

\section{APPENDIX G \\ PROOF OF THEOREM 7}

Lemma $G$ : Let $\mathbf{H}$ be an $n \times M$ random matrix with $M$ mutually independent columns $\mathbf{h}_{1} \cdots \mathbf{h}_{M}$, and $\boldsymbol{\lambda}$ is an $M \times M$ diagonal matrix with elements $\Lambda_{k k}=n^{-1}\left\|\mathbf{h}_{k}\right\|^{2}$, where $\mathbf{h}_{k}, k=1 \cdots M$, is a Gaussian circularly symmetric vector with the correlation matrix $\mathbf{R}_{k}=E\left\{\mathbf{h}_{k} \mathbf{h}_{k}^{H}\right\} . \mathbf{H}^{H} \mathbf{H} / n$ and $\boldsymbol{\lambda}$ are asymptotically equivalent in probability, i.e., $\mathbf{H}^{H} \mathbf{H} / n \stackrel{p}{\rightarrow} \boldsymbol{\lambda}$ as $n \rightarrow \infty$, if $n^{-1} \operatorname{tr}\left\{\mathbf{R}_{k}\right\}<\infty$ and $n^{-2} \operatorname{tr}\left[\mathbf{R}_{k} \mathbf{R}_{m}\right] \rightarrow 0, k, m=1 \cdots M$, as $n \rightarrow \infty$.

Proof: Without loss of generality, assume that $\mathbf{h}_{k}, k=1 \cdots M$ are normalized, so that $n^{-1} \operatorname{tr}\left\{\mathbf{R}_{k}\right\}=1$. Thus $E\left\{\mathbf{H}^{H} \mathbf{H} / n\right\}=$ $E\{\boldsymbol{\lambda}\}=\mathbf{I}$, where $\mathbf{I}$ is the $M \times M$ identity matrix, since $\mathbf{h}_{1} \cdots \mathbf{h}_{M}$ are mutually independent. From Chebyshev inequality [24], for any $\varepsilon>0$

$$
\operatorname{Pr}\left\{\left\|\mathbf{H}^{H} \mathbf{H} / n-\boldsymbol{\lambda}\right\| \geq \varepsilon\right\} \leq \varepsilon^{-2} \cdot E\left\{\left\|\mathbf{H}^{H} \mathbf{H} / n-\boldsymbol{\lambda}\right\|^{2}\right\}
$$

where

$$
\begin{aligned}
E\{\| & \left.\mathbf{H}^{H} \mathbf{H} / n-\boldsymbol{\lambda} \|^{2}\right\} \\
= & n^{-2} \operatorname{tr}\left[E\left\{\mathbf{H}^{H} \mathbf{H} \mathbf{H}^{H} \mathbf{H}\right\}\right] \\
& -2 \operatorname{tr}\left[E\left\{\mathbf{H}^{H} \mathbf{H} / n \cdot \boldsymbol{\lambda}\right\}\right]+\operatorname{tr}[E\{\boldsymbol{\Lambda} \boldsymbol{\Lambda}\}] \\
= & n^{-2} \operatorname{tr}\left[E\left\{\mathbf{H} \mathbf{H}^{H} \mathbf{H} \mathbf{H}^{H}\right\}\right]-\operatorname{tr}[E\{\boldsymbol{\Lambda} \boldsymbol{\Lambda}\}] \\
= & n^{-2} \sum_{m=1}^{M} \sum_{k=1}^{M} \operatorname{tr}\left[E\left\{\mathbf{h}_{m} \mathbf{h}_{m}^{H} \mathbf{h}_{k} \mathbf{h}_{k}^{H}\right\}\right] \\
& -n^{-2} \sum_{m=1}^{M} \operatorname{tr}\left[E\left\{\mathbf{h}_{m} \mathbf{h}_{m}^{H} \mathbf{h}_{m} \mathbf{h}_{m}^{H}\right\}\right] \\
= & n^{-2} \sum_{m=1}^{M} \sum_{\substack{k=1 \\
k \neq m}}^{M} \operatorname{tr}\left[E\left\{\mathbf{h}_{m} \mathbf{h}_{m}^{H} \mathbf{h}_{k} \mathbf{h}_{k}^{H}\right\}\right] \\
= & n^{-2} \sum_{m=1}^{M} \sum_{\substack{k=1 \\
k \neq m}}^{M} \operatorname{tr}\left[\mathbf{R}_{m} \mathbf{R}_{k}\right]
\end{aligned}
$$

where the second equality is since $\operatorname{tr}\left[E\left\{\mathbf{H}^{H} \mathbf{H} / n \cdot \boldsymbol{\lambda}\right\}\right]=\operatorname{tr}[E\{\boldsymbol{\Lambda} \boldsymbol{\Lambda}\}]$. By substituting (G2) in (G1)

$$
\operatorname{Pr}\left\{\left\|\mathbf{H}^{H} \mathbf{H} / n-\boldsymbol{\lambda}\right\| \geq \varepsilon\right\} \leq \frac{1}{(\varepsilon \cdot n)^{2}} \sum_{m=1}^{M} \sum_{\substack{k=1 \\ k \neq m}}^{M} \operatorname{tr}\left[\mathbf{R}_{m} \mathbf{R}_{k}\right]
$$

Therefore, if $n^{-2} \operatorname{tr}\left[\mathbf{R}_{k} \mathbf{R}_{m}\right] \rightarrow 0, k, m=1 \cdots M$, as $n \rightarrow \infty$, then from (G3) using the continuity property of the probability measure, for any $\varepsilon>0$

$$
\operatorname{Pr}\left\{\left\|\mathbf{H}^{H} \mathbf{H} / n-\lambda\right\| \geq \varepsilon\right\} \rightarrow 0, \text { as } n \rightarrow \infty
$$

Q.E.D.

Proof of Theorem 7: From Lemma G, $\mathbf{B}_{t} \stackrel{p}{\rightarrow} \boldsymbol{\lambda}_{t}$ and $\mathbf{B}_{r} \stackrel{p}{\rightarrow} \boldsymbol{\lambda}_{r}$ where $\boldsymbol{\lambda}_{t}$ and $\boldsymbol{\lambda}_{r}$ are diagonal matrices with elements $n_{t}^{-1}\left\|\mathbf{h}_{t k}\right\|^{2}$ and 
$n_{r}^{-1}\left\|\mathbf{h}_{r M}\right\|^{2}, k, m=1 \cdots M$, respectively. Thus, from Slutsky Theorem [30, Th. 6'a], as both $n_{t}, n_{r} \rightarrow \infty$

$$
\begin{aligned}
C \stackrel{p}{\rightarrow} \ln \operatorname{det}\left(\mathbf{I}+\gamma_{0} \boldsymbol{\lambda}_{r} \mathbf{A} \mathbf{\Lambda}_{t} \mathbf{A}^{H}\right) \\
=\sum_{k=1}^{M} \ln \left(1+\frac{\left|a_{k}\right|^{2} \gamma_{0}}{n_{t} n_{r}}\left\|\mathbf{h}_{t k}\right\|^{2}\left\|\mathbf{h}_{r k}\right\|^{2}\right)
\end{aligned}
$$

Q.E.D.

\section{ACKNOWLEDGMENT}

The authors would like to thank anonymous reviewers for valuable comments and suggestions.

\section{REFERENCES}

[1] I. E. Telatar, "Capacity of multiantenna Gaussian channels," AT\&T Bell Labs, Internal Tech. Memo, Jun. 1995.

[2] G. J. Foschini and M. J. Gans, "On limits of wireless communications in a fading environment when using multiple antennas," Wireless Pers. Commun., vol. 6, no. 3, pp. 311-335, Mar. 1998.

[3] M. Chiani, M. Z. Win, and A. Zanella, "On the capacity of spatially correlated MIMO rayleigh-fading channels," IEEE Trans. Inf. Theory, vol. 49, no. 10, pp. 2363-2371, Oct. 2003.

[4] G. Levin and S. Loyka, "Statistical approach to MIMO capacity analysis in a fading channel," in Proc. 2004 IEEE Veh. Technol. Conf. VTC2004-Fall, Sep. 2004, vol. 3, pp. 1548-1552.

[5] L. Musavian, M. Dohler, M. R. Nakhai, and A. H. Aghvami, "Closedform capacity expressions of orthogonalized correlated MIMO channels," IEEE Commun. Lett., vol. 8, no. 6, pp. 365-367, Jun. 2004.

[6] M. Dohler and A. H. Aghvami, "Information outage probability of distributed STBC's over nakagami fading channels," IEEE Commun. Lett., vol. 8, no. 7, pp. 437-439, Jul. 2004.

[7] D. Chizhik, G. J. Foschini, and R. A. Valenzuela, "Capacities of multielement transmit and receive antennas: Correlations and keyholes," Electron. Lett., vol. 36, no. 13, pp. 1099-1100, Jun. 2000.

[8] D. Chizhik, G. J. Foschini, M. J. Gans, and R. A. Valenzuela, "Keyholes, correlations and capacities of multielement transmit and receive antennas," IEEE Trans. Wireless Commun., vol. 1, no. 2, pp. 361-368, Apr. 2002

[9] D. Porrat, P. Kyritsi, and D. C. Cox, "MIMO capacity in hallways and adjacent rooms," IEEE Globecom'02, vol. 2, pp. 17-21, Nov. 2002.

[10] P. Almers, F. Tufvensson, and A. F. Molisch, "Measurements of keyhole effect in a wireless multiple-input multiple-output (MIMO) channel," IEEE Commun. Lett., vol. 7, no. 8, pp. 373-375, Aug. 2003.

[11] P. Almers, F. Tufvensson, and A. F. Molisch, "Keyhole effect in MIMO wireless channels: Measurements and theory," IEEE Trans. Wireless Commun., vol. 5, no. 12, pp. 3596-3604, Dec. 2006.

[12] H. Shin and J. H. Lee, "Capacity of multiple-antenna fading channels: Spatial fading correlation, double scattering and keyhole," IEEE Trans. Inf. Theory, vol. 49, no. 10, pp. 2636-2646, Oct. 2003.

[13] H. Shin and J. H. Lee, "Performance analysis of space-time block codes over keyhole nakagami-m fading channels," IEEE Trans. Veh. Technol., vol. 53, no. 2, pp. 351-362, Mar. 2004.

[14] X. W. Cui and Z. M. Feng, "Lower capacity bound for MIMO correlated fading channels with keyhole," IEEE Commun. Lett., vol. 8, no. 8, pp. 500-5002, Aug. 2004.

[15] S. Loyka and A. Kouki, "On MIMO channel capacity, correlations, and keyholes: Analysis of degenerate channels," IEEE Trans. Commun., vol. 50, no. 12, pp. 1886-1888, Dec. 2002.

[16] B. M. Hochwald, T. L. Marzetta, and V. Tarokh, "Multiple-antenna channel hardening and its implications for rate feedback and scheduling," IEEE Trans. Inf. Theory, vol. 50, no. 9, pp. 1893-1909, Sep. 2004.

[17] C. Martin and B. Ottersten, "Asymptotic eigenvalue distributions and capacity for MIMO channels under correlated fading," IEEE Trans. Wireless Commun., vol. 3, no. 4, pp. 1350-1358, Jul. 2004.

[18] S. Loyka, "Channel capacity of MIMO architecture using the exponential correlation matrix," IEEE Commun. Lett., vol. 5, no. 9, pp. 369-371, Sep. 2001.
[19] F. Adachi et al., "Crosscorrelation between the envelopes of $900 \mathrm{MHz}$ signals received at a mobile radio base station site," Inst. Elect. Eng. Proc., vol. 133, no. 6, pp. 506-512, Oct. 1986.

[20] T. S. Chu and L. J. Greenstein, "A semi-empirical representation of antenna diversity gain at cellular and PCS base stations," IEEE Trans. Commun., vol. 45, no. 6, pp. 644-646, Jun. 1997.

[21] A. Paulraj, R. Nabar, and D. Gore, Introduction to Space-Time Wireless Communications. Cambridge, U.K.: Cambridge Univ. Press, 2003.

[22] S. M. Alamouti, "A simple transmit diversity technique for wireless communications," IEEE J. Sel. Areas Commun., vol. 16, no. 8, pp. 1451-1458, Oct. 1998.

[23] W. C. Jakes, Microwave Mobile Communications. New York: Wiley, 1974.

[24] D. Zwillinger, CRC Standard Mathematical Tables and Formulae, 31st ed. Boca Raton, FL: CRC, 1996.

[25] H. Boche and E. A. Jorswieck, "On the ergodic capacity as a function of the correlation properties in systems with multiple transmit antennas without CSI at the transmitter," IEEE Trans. Commun., vol. 52, no. 10, pp. 1654-1657, Oct. 2004.

[26] A. W. Marshall and I. Olkin, Inequalities: Theory of Majorization and Its Application. London, U.K.: Math. Sci. Eng., 1979, vol. 143, Academic.

[27] G. Levin and S. Loyka, "Comments on asymptotic eigenvalue distributions and capacity for MIMO channels under correlated fading," IEEE Trans. Wireless Commun., vol. 7, no. 2, pp. 475-479, Feb. 2008

[28] S. Loyka and J. Mosig, "Channel capacity of n-antenna BLAST architecture," Electron. Lett., vol. 36, no. 7, pp. 660-661, Mar. 2000.

[29] A. M. Mathai and S. B. Provost, Quadratic Forms in Random Variables. New York: Marcel Dekker, 1992.

[30] T. S. Ferguson, A Course in Large Sample Theory, 1st ed. London, U.K.: Chapman \& Hall/CRC, 2002, Reprint.

[31] H. Cramer, Mathematical Methods of Statistics. Princeton, NJ: Princeton Univ. Press, 1966, 11th printing.

[32] B. V. Gnedenko, The Theory of Probability, 4th ed. New York: Chelsea, 1968.

[33] S. Loyka and F. Gagnon, "V-BLAST without optimal ordering: Analytical performance evaluation for rayleigh fading channels," IEEE Trans. Commun., vol. 54, no. 6, pp. 1109-1120, June 2006.

[34] R. M. Gray, "On the asymptotic eigenvalue distribution of Toeplitz matrices," IEEE Trans. Inf. Theory, vol. IT-18, no. 6, pp. 725-730, Nov. 1972.

[35] İ. E. Telatar, "Capacity of multi-antenna Gaussian channels," Europ. Trans. Telecommun., vol. 10, no. 6, Dec. 1999. 